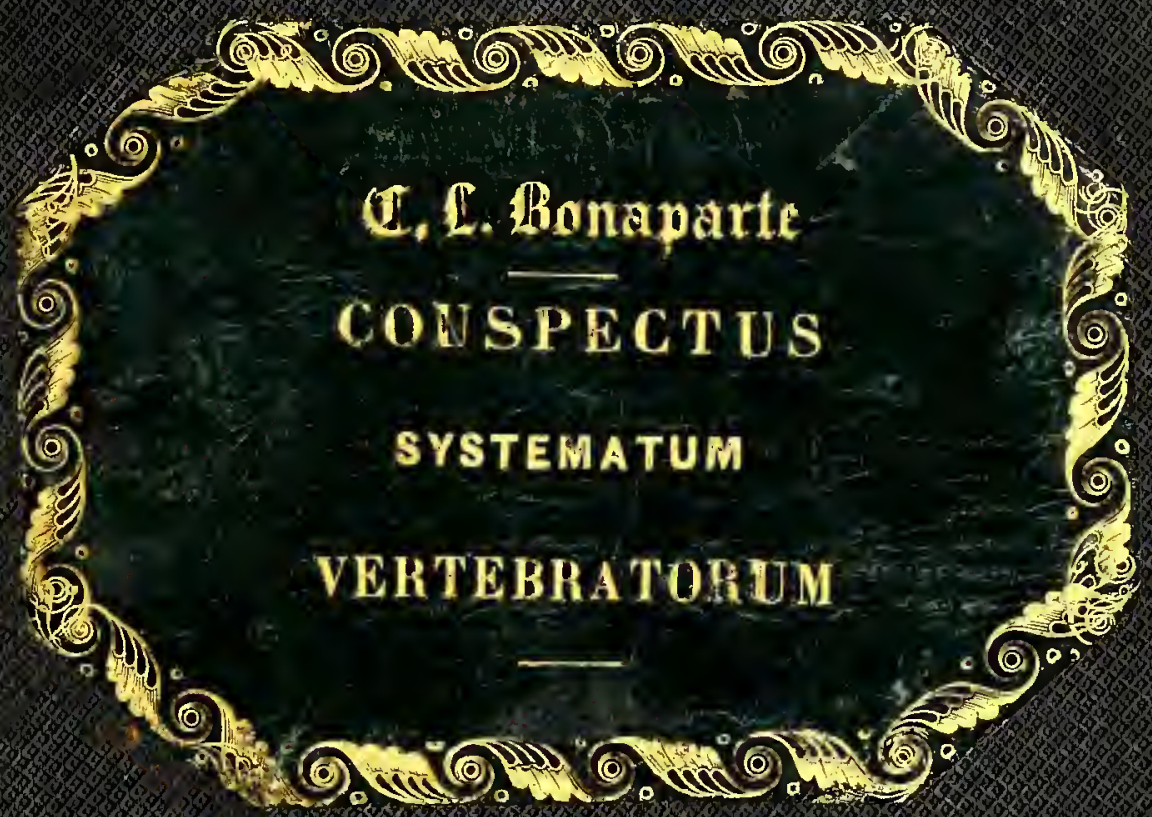


Digitized by the Internet Archive in 2011 with funding from

University of Illinois Urbana-Champaign

http://www.archive.org/details/newsystematicarro0bona 
XVI. A New Systematic Arrangement of I'ertebrated Animals. By C. L Bonaparte, Prince of Musignano, F.M.L.S., Sic.

Read November 7th, 1837.

Primam Regni animalis Provinciam constitumnt Animalia Vertebrata: sceleto interno custodienti cerebrum stipitemque nervorum sub cranio et rertebris costas artuunque ossa a musculis protecta regentibus, visceribus cæeteris cavitate trunci conclusis. Vása in apparatu continuo: cor musculare: sanguis ruber: maxilla supra mandibulam incumbens: quinorum organa sensunm, Lingua, Nares, Oculi, Aures, Papillæe prina quatuor in facjei cavitatibus distincta, ad gustum, olfactum, visum et anditum. Artus non plures quam quatuor. Sexus distincti.

\section{Classium Distinctio.}

1. MAMMALIA. Sanguis calidus: pulmones liberi: manmæ. Vivipara.

2. AVES. Sanguis calidus : pulmones affixi: alæ. Ovipara.

3. AMPHIBIA. Sanguis frigidus: pulmones liberi. Ovipara vel Ovovivipara.

4. PISCES. Sanguis frigidus: pulmones nulli: branchiæ. Ovipara rel Ovovivipara.

\section{Classis I. MAMMALIA.}

Animalia vertebrata, sanguine calido, circulatione duplici perfecta, vivipara, pullos lactantia : pulmones bini, imperforati, in pectoris cavitate suspensi : cor' biloculare, biauritun : dentes fere in omnibus: corpus utplurimum pilosum : artus, vix paucis exceptis, quatuor manifesti : collo caput fere semper distinctum a trunco.

vol. XVIII. 


\section{Ordinum Distributio.}

\section{Series 1. PLACENTALIA.}

Generationis organa ab ano exterius disereta: vagina uniforis: fota matura: mamma conspicux: ossula ad pubem accessoria nulla: scrotum peni postpositum.

\section{Subclassis 1. EDUCABILIA.}

Cerebrum bi- (v'el tri-) lobum*.

1. Prinates. (Quadrumana). Artus quatuor, antici manibus terminati : ungnes digitorum apices tantum obtegentes: triplex dentium qualitas; incisivi superiores 2 rel 4; molares tritorii : manmæ pectorales : penis liber, pensilis.

2. Fere. (Camivora.) Artus quatuor liberi, exporrecti, distincti, gradientes, ungues digitorum apices tantum obtegentes: triplex dentiun qualitas; molares trimorphi, antici sectorii, postici sine tuberculis acutis; ferinus utrinque saltem unus, laniarii duo validi, et incisivi sex in utraque maxilla: mamme abdominales: penis inclusus vagina abdomini adlizente.

3. Prisipeda. (Imphibia.) Artus quatuor, brevissimi, retracti, obvoluti, pinniformes: postici longiores, reversi, invicem proximi : ungues digitornm apices tantum obtegentes: triplex dentiun qualitas: namma abloninales: penis vagina abdomini adharente inclusus.

4. CeTE. (Natantia.) Artus dno, posticis nullis, pinnifornes: caput deficiente collo indistinctum : corpus pisciforme, caudaque ligamentosa horizontali pinniformi terminatmu. Aquatica; auriculis, pilisque destitutu.

5. 13ellue. (Pachydermata.) Artus quatuor; claviculae nullac; antibrachium constanter pronum: ungues sculponei, digitorum phalanges cxtremas obvolventes: dentiun qualitas sapius triplex: stomachus simplex, ant licet compositus rmminationis impotens.

6. Pecora. (Ruminuntia.) Artus quatuor, bisulci; clavicule nulle; antibrachimm constanter pronum : ossa metacarpi et metatirsi connata : ungues

A constructione cerebri, scilicet a lohorum, quibus organum illud nobilissimum constet, quantitate, Mammalium distributionem laudabiliter peti posse didicimus in Museo naturali Lugdunensi, cui meritissime præest cl. Jourdanus, qui jam diu animale Regnum juxta nervorum vitalissimam conditionem examinandum suscepit. Confectum hujusmodi Systema quam primum in lucem proferri nemo est qui non cupiat. Divisionem vero in Placentalia atque Oroviripara, etsi ab aliis adumbratam, certis limitibus hodie conclusam cl. Oweno, Anglo, debemus. 
sculponei, digitorum phalanges extremas obvolventes: dentium qualitas raro triplex: ventriculis quatuor ruminantia.

Cerebrum unilobum.

Subclassis 2. INEDUCABILIA.

7. Bruta. (Edentata.) Artus quatuor liberi; ungues digitorum summitaten obvolventes, conici, fere sculponei : dentes radicibus destituti, aut duplicis aut unicæe qualitatis, aut nulli; incisivi nulli; ubi molares 14-98.

8. Chemroptera. (Volit ntia.) Artus quatuor, antici, digitis longissimis (dempto brevissimo pollicc), membrana nuda ad pedes usque producta aliformi conjunctis : ungnes digitorun apices tantum obtegentes: triplex dentium qualitas, incisivi superiores $0-2-4$ : mammæ duo pectorales: penis liber, pensilis. Nocturna.

9. Bestiæ. (Insectivora.) Artus quatuor liberi, manibus non terminati: ungues digitorum apices tantum obtegentes: triplex dentium qualitas; molares dimorphi, ferinis nullis; antici spurii, postici tuberculis acutis pluribus coronati, supra subtusque hinc inde quatuor: incisivi 2-6: mammæ plures abdominales: penis vagina abdomini adhærente inclusus.

10. Gunes. (Rosores.) Artus quatuor: ungues digitorum apices tantum obtegentes: duplex dentium qualitas, laniariis nullis; incisivi infra supraque dno, elongati, superioribus quandoque duo accessorii additi: molares ad summum 24, tritorii : mandibulis horizontaliter promotis rosores.

\section{Series 2. OVOVIVIPARA.}

Generationis organa ab ano cxterius haud discreta: fœeta abortiva, extra uterum maturanda: mamma inconspicuæ: ossula ad pubem duo accessoria : scrotum prapositum peni retroverso, aut nullum.

11. Marsupialia. (Didelpha.) Artus quatuor, gradientes, postici sape manibus terminati: ungues digitorum apices tantum obtegentes: dentes alveolares, duplicis aut triplicis qualitatis: foninarum manmæ marsupio abdominali, vel ejus rudimentali plica, abscondita: vagina biforis.

12. Monotremata. (Reptantia.) Artus quatuor, aut natatores ant fossores: ungues digitorum apices tantum obtegentes: dentes alveolares nulli: cloaca excretionis sirnul ac generationis organa intra se continens: narsupium abdominale nullum: vagina uniforis. 
Contrabendo nunc chatacteres adeo ut mo oculi ictu inspiciamus qualem Mammalium classis distributioncm obtineat meliorem ex antea scriptis, tabellam hanc simplicissimam cxatavimus.

\section{MAMMALIA. \\ Series 1. PLACENTALIA.}

Subclassis 1. EDUCABILIA.

1. Primates. Altus antici manus.

2. Ferk. Molares sectorii, canini validi.

3. Pinnipedin. Artus pinniformes.

4. Cete. Corpus pisciforme.

5. Bellua. Ungulata, haud ruminantia.

6. Pecora. Ungulata ruminantia.
Subclassis 2. INEDCCABILIA.

7. Bruta. Subungulata, imperfecte dentata.

8. Cheiroptera. Artus antici aliformes.

9. Bestre. Molares cuspidati.

10. Gures. Incisivis elongatis rosoria.

Series 2. OTOTIITPARA.

11. Marsuphata. Mamme occultre.

12. Monotremita. Cloací.

Conspectus Famillarum et Sebfaniliarum.

Series 1. PLACENTALIA.

Subclassis 1. EDUCABILIA.

Ordo 1. PRIMLTES.

1. Honnids. Artunm tantum antici in manus desinentes pollice crique digito opponibili.

1. Hominima. Corpus crectum, plantigradum, ecandatum.

2. Smide. Artus singuli in manus desinentes, pollice, saltem in posticis, cui- 
que digito opponibili : dentes ineisivi plus minus erecti infra supraque quatuor: vultus denudatus. Anthropomorpha.

2. Simina. Manus singulæ pollice cuique digito opponibili: dentes infia supraque hinc inde quinque tuberculati : nares approximate: ungues breves, depressi.

3. Cebina. Manus singule, anticis interdum imperfectis, pollice cuique digito opponibili: dentes molares infra supraque hine inde sex, tuberculati: nares inter se remotie: ungues breves, depressi.

4. Hapalina. Manus tantum postica pollice cnique digito opponibili: dentes molares infra supraque hine inde quinque, enspidati: nares inter se remote: ungues longissimi, arcuati, compressi, acuti.

3. Lemurid.e. Artus singuli in manus desinentes, pollice cuique digito opponibili : dentes incisivi procumbentes, aut supra rel infra plusquam quatuor: molares cuspidati: vultus pilosus: nares terminales, sinuosie. Feriformia.

5. Lemurina. Artus caudaque liberi.

6. Galeopithecina. Artus antici membrana villosa cum posticis caùdaque conjuncti.

\section{Ordo 2. FER E.}

4. Cercoleptidide. Mammæ duo tantum, inguinales: lingua longissima, extensilis: cauda prehendens, tota hirsuta.

7. Cercoleptidinu. Dentes 36, sex nempe incisiv:, duo laniarii, molares decem, spuriis 4, in utraque maxilla. Anomala. Primates firgivoros cum Bestiis conjungit.

5. Urside. Dentes molares postici tritores: pedes plantigraldi, plantis denudatis: ungues obtusiculi.

8. Ursina. Dentes incisivi mandibulie ad lineam collocati : ungues scansorii.

9. Melina. Dentes incisivi mandibula extra lincam collocati: ungues fossores.

6. Feld de. Dentes molares posteriores, demptis postremis minoribus, sectorii : pedes plerunque digitigradi, plantis pilosis: ungues acntissimi.

10. Viverrina. Dentes molares tuberculati utrinque bini post carnivorum supra, infra unus: lingua aspera: folliculus glandulosus pone anum. 
11. Camina. Dentes molares tuberculati utrinque bini post carnivorun infra supraque: lingua lovis.

12. Felina. Dentes molares tuberculati unlli in mandibula: lingua asper"a.

13. Mustelina. Dentes molares tuberculati in utraque maxilla: unus post carnivorum utrinque supra: lingua læris: pedes congrui : cauda tereticula. Corpus elongatm, gracile, alnctile: pedes breves.

14. Lutrimu. Dentes molares tuberculati, utrinque unus post carnivorum infra supraque: lingua seabricula: pedes subretracti, subobvoluti, postici laterales podio antror'sun verso: cauda applanata.

\section{Ordo 3. PINTIPEDIA.}

7. Phocus. Dentes laniarii mediocres, inclusi : mammae duse ventrales.

15. Otarina. Auricula prominula: dentes incisivi superiores bicultres, inferiores bicuspides : digiti palmanum inmobiles; mngues plani.

16. Plocina. Auricula vix ullæ: dentes incisivi acuti: digiti singuli unobiles, ungues acuti.

8. 'Truchechidæ. Dentes laniarii longissimi, producti, validi, in maxilla tantmm: mamma quatmol ventrales.

17. Trichechina. Auricule mullæ: rostrum brevissinum, obtusum: nares supere: dentes incisivi smpra dno minuti, infra nulli. Corpms obesmm.

\section{Ordo 4. CETE.}

?. Manatide. Dentes molares compositi aut senni-compositi, corona plana ant sulcata: mamme pectorales: spiracula nulla: intestinum caecum. Phytophaga.

18. Manutinu. Artus fere brachiiformes, plerumque mnguiculati.

(0. Delphinde. Dentes simplices, coniei : artus prorsus pinniformes, exunguiculati: mamma inguinales: spiracula: caput vel mediocre vel palrum: caecum nullum. Zoophaga.

19. Delphimina. Dentes sxpius unmerosi infra supraque.

20. Monodontina. Dentes tantum dno, praelongi, acnti, ex tortili fabrica, osse maxillari infixi (uno sapius abortivo).

11. Phrseternde. Dentes numerosi, simplices, conici: artus prorsus pinnifolmes, exunguiculati : caput immane: cacum nullum ? 
21. Physeterina. Dentes inferiores a totidem maxillæ foreis excipiendi: superiores parvuli, absconditi.

12. BaLenide. Dentes nulli: cæcmin parvum.

22. Balanina. Laminæ corneæ binæ in maxilla inæqualiter pectiuatie ve hinc inde oceludentes loco dentinu.

Ordo 5. BELLUE.

13. Esefhantide. Digiti sub tegumentis reconditi, ungue tantum dignoscendi.

23. Dinotherina. Pedes pentadactyli: dentes molares coronide transversim sulcata; incisivi infra duo prelongi, deflexi.

24. Elephantina. Pedes pentadactyli: dentes molares utrinque bini maximi, lamellosi ant manillosi : nasus proboscideus.

14. SuIDæ. Digiti ad apicem saltem fissi.

25. Hippopotamina. Pedes tetradactyli: dentes molares complicati, nnmerosi : chilona latum, obtnsum.

26. Rhinocerontina. Pedes tridactyli: dentes molares complicati, numerosi : nasus corniger.

27. Trapirina. Pedes antici tetradactyli, postici tridactyli: digiti cute obvoluti, ad apicem fissi : nasus productus, mobilis.

28. Suina. Pedes tetradactyli, postici interdum tridactyli : digiti insessores constanter dwo: nasus subproboscideus.

29. Anoplotherina. Pedes didactyli.

15. Hyracid.e. Digiti artuum anticorum quatuor, posticorum tres, ommes cute obvoluti, apice fissi : ungues lamellares.

30. Hyracina. Digitus artumm posticormu internus ungue curvo munitu: cutis dense jilosa: dentes incisivi supra duo. Gliribus accedentiu.

16. EQuida. Pedes tridactyli duobus digitis abortivis lateralibus absconditin: principalis solida ungula couvallatus.

31. Equinu. Corjus dense pilosum, collo candaque longe crinitum: dentes incisivi infra supraque sex.

Ordo 6. PECORA.

17. Canelide. Dentes laniarii infra supraque: duo incisivi supra, infra sex: cornua nulla. 
32. Ciemelina. Rostrum productum: sinus lacrymales nulli : pedes subtus callosi, digitis cute obrolutis, solo apice bisulei.

18. Cervom. Dentes limiarii infra nulli; incisivi supra nulli, infra octo: copnua in maribus fere ordinaria, rarissima in fominis, caduca, solidi, pelumenlata, ramosa, cuticula villosa, temporaria saltem, induta.

33. Moschina. Dentes laniarii duo produeti, supra in maribus: simus lacrymales nulli : cornua nullit: folliculus preputialis moschifer:

34. Covino. Dentes laniarii plerumpue nulli: sinus lacrymales sapius magni : cornua in maribus.

19. Camblopardalide. Dentes lamiarii nu!li: incisivi supra nulli, infra octo: cornua in utrogue sexu, peremuia, solida, brevia, simplicia. cuticula villosa induta.

35. Cumelopardulino. Dentes molares utringue sex contigui : pedes prorsus bisulci.

20. Bovide. Dentes lanialii nulli : incisivi supra nulli, infra octo: cornua peremia, sxpius in utroque sexu, ex osse frontali producta, elastico tegumento vaginata.

36. Antilopina. Cornua solida.

37. Borina. Cormua carernosa.

\section{Subclassis 2. INEDUCABILIA.}

$$
\text { Ordo } r \text {. BRLT. }
$$

21. Mrmecophagibx. Dentes mulli: os perexigumu: lingua angusta, emissilis.

38. Myrmecophagina. Corpus pilosum: ungues antici ralidi, margine acuto.

:39. Manidina. Corpus sruamosum.

22. Dassupdo.e. Dentes: laurarii nulli: molares 26-98: rostrum productum.

40. Dasypodinct. Corpus cataphractum.

41. Orycteropodina. Corpus pilosum.

23. BRanpodide. Dentes: incisivi nulli: molares non ultra 18: rostrum breve: artus antici longiores. 
42. Bralypodina. Corpus villosum: dentes laniarii aenti: mamma duo, pectorales! digiti cute juncti: ungues maximi, falculares.

\section{Orio s. CIIEIROPTERA.}

24. Pteropodide. Dentes molares, aut obtuse tubereulati, ant læves; incisivi parri, inanes, inter validos laniarios stipati: digitus index omniun phalangium mumero absolutus, ungniculatus. Frugivora, Gregaria.

43. Pteropolima. Nasus sinplex: nares tubulosæ: labia tenuia: tragus mullus : caput conicum : unguis digiti indicis plerumque acutus: membrana interfemoralis brevissima: cauda vel brevissima vel nulla.

25. Vespertulonida. Dentes molares tuberculis acutis coronati : nullus alaris digitus omni phalangium numero absolutus: index exunguiculatus. Insectivora.

44. Noctilionima. Nasus simplex: labia magna, prolapsa: cauda brevis, crassa, apice libera.

45. Tespertilioninu. Nasus simplex: labia congrua: cauda longa, membrana interfemorali aupla obvoluta.

46. Rhinolophima. Nasus appendice foliacea complicata: tragus unllus.

47. Rhinopomina. Nisus appendice foliacea simplici : tragus distinetus.

26. VAmprride. Dentes molares tuberenlis acutis roronati : tertius tantun alaris digitus omni phalangium mumero absolutus : index exunguiculitus. Insectirorn.

48. Iumpyrina. Nisus appendice foliacea simplici: tragus distinctus.

Ordo 9. BESTIA.

27. Thlpide. Altus antici fossoles.

49. Talpimu. Rostrum productum.

29. Soricine. Artus singuli vel ambulatores rel natatores : cutis pilosa.

50. Macroscelididina. Rostrum proboscideum: dentes incisivi infra quatmor, supra sex; canini mulli: artus postici anticis valde lougiores.

51. Soricina. Rostrum attenuatum: dentes incisivi infra suprague duo; canini muli : auricula: pedes fissi.

52. Myogalina. Rostrum proboscideum: dentes incisivi infra quatuor, duo supra; canini mulli: anriculæ nulla: pedes palmati.

VOL. XVIII.

211 
53. Cladobatidima. Rostrum elongatum : dentes incisivi infua sex, quatuor supı ; canini mulli : artus aequilongi: ungues adunci, acutissimi.

29. Erivacende. Artus singuli ambulatores: cutis spinosa.

54. C'entetiua. Corpus haud conglobabile: dentes canini.

55. Erinaceimu. Corpus conglobabile: cauda brevissima: dentes canini nulli.

Ordo 10. GLIRES.

30. Muride. Claviculæ perfectæ: dentes molares simplices.

56. Echymydina. Cauda squamata: vellus aculeis mixtum.

57. Murina. Cauda squamata: vellus setis mixtum.

58. Dipodina. Cauda longissima, apice floccifero : pedes saltatorii, antici

- breves, postici longissimi.

59. Sciurime. Cauda longa, villosa, sepius disticha: rellus molle, uniforme: pedes aequilongi.

60. Arctomydiua. Canda vel brevis vel nulla: vellus molle, subuniforme: pedes iequilongi.

61. Aspalucina. Cauda Jnevis: vellus loude: pedes breves; anticorum ungucs fossores: oculi auresque exigui : dentes incisivi praelongi, manifesti.

31. Castonude. Clavicula perfecta: dentes molares compositi.

62. Arreolima. Dentes molares radicibus destituti, lamellosi. Herbivora.

63. Castorina. Dentes molares radicilus instructi.

32. Cheirourdide. Clavicula perfectae: mammae duo, inguinales : cauda longissima.

64. Cheirmydina. Pedes pentadactyli, digito medio clongato, gracillino, uudo: postiei desinentes in manus polliee unğue laminari cuique digito opponibili.

33. Hrstricida. Clavicule imperfectre: corpus spinosum; dentes incisivi duo supra: pedes antici tetradactyli, postici pentadactyli.

65. Hystriciuu. Dentes molares coronide plana sublamellosi : lingua hispida.

34. Leporme. Clavienle imperfectre: corpus pilosum : dentes incisivi supra quatuor (in junioribus sex): pedes antici tetralactyli, postici pentadictyli. 
66. Leporina. Corpus plantreque pilosa: dentes molares límellosi.

35. Lugostomde. Clavicula imperfecta : corpus mollissime, uniformiter villosmm : dentes incisivi duo supra: pedes antici breves, postici elongati.

67. Lagostomina. Dentes incisivi inferi canaliculati : cauda pectinata.

36. Cavide. Claviculae imperfecte : corpus pilosun : dentes incisivi duo supra; molares scxdecim: pedes postici, vel tridactyli, vel pentadactyli, utroque digito laterali minimo.

68. Cavina. Dentes molares radicibus destituti, lamellosi.

69. Dasyproctina. Dentes molares compositi.

\section{Series 2. OVOVIVIPARA.}

Ordo 11. MARSUPIALIA.

37. Halmaturide. Dentes in modum plus minus Glirmm: incisivi elongati; carnivori nnlli : molares tuberculis coronati.

70. Phascolomydina. Dentes in modum penitus Glirum: incisivi elongati infia supraque duo ; laniarii nulli, vel tantum supra, exigui; molares tuberculis transversis dnobus: caput grande, depressum : artus breves: ungues fosşores: canda nulla.

71. Halmaturina. Dentes incisivi duo infra longi, lati, acuti, sex supra: laniarii infra saltem nulli: artus antici brevissimi, postici longissimi, digitis duobus conjunctis, pollice nullo: cauda fulciens.

72. Petaurina. Dentes incisivi duo infra longi, lati, acuti, sex supra: laniarii longi, acuti supra ; exiles, latentes infra, vel nulli : artus æquilongi digitis duobus conjunctis, pollice grandi, exungui, fere retroverso: canda prehendens.

38. Didezphide. Dentes in modun Bestiarum : ferini nulli: molares tubereulis acutis coronati utrinque tres rel quatuor.

73. Didelphina. Artus postici in mamus pollice cuique digito opponibili desinentes: canda prehendens partim muda: dentes incisivi decem stipra, infra octo: lingma hispida.

39. Thy Lacinide. Dentes in modum Ferarum : infra supraque carnivori quatuor. 
74. Thulacimina. Artus postici pollice nullo: caucla pilosa: dentes 46. Ommium. Ferarum ipsissimarmm magis camivora ratione dentium.

\section{Ordo 12. MONOTREMLTA.}

40. Echinxida. Corpus spinosum : rostrum cylindracenn, ittenuatum : pedes fossores.

75. Echidnina. Aculei parvi palato affixi loco dentium: lingua emissilis.

41. Orxithorhychum. Colpus pilosum: rostrum ralcle depressum, latum (anatinum): peeles paluati.

76. Ornithorhynchina. Dentes molares utrinque duo infra supraque: lingual lata, mollis, carnosa.

\section{ClaAsis II. ATES.}

Animalia vertebrata, sanguine calido, circulatione duplici, ovipara, rolatilia: pulmones bini indivisi, cribrosi, costis adherentes : cor biloculare, biauritum : rostrum colneum, dentibus destitutum : corpus plumosum : sternum fere in ommibus carinatum : os furcule: ala pedesque duo.

\section{Ordinum Distributio}

\section{Subclassis 1. INSESSORES.}

Digitus posticus (pollex, sive digitus externus retrorersus) endem plano ac antici insertus. totus sulo insistens, constrietor. Homogame fore omnes: pullomm inbecillum altrices.

* Distribuere arium classem in plures quam octo ordines naturales conabar. Inutilis sane labor, a quo discessi. Quis enim Accipitres cum creteris potius Insessoribus conjungendos, quis Gallinas in plures ordines discindere audeat? Anseres quidem in quatuor distribuerentur facile, in Anseres genuinas videlicet, in Pelectmos, in Gariss, in Crinutores: Grallurum vero (Struthionibus demptis) subdirisio alia omnino non ubtinet. Si Passerum inde spectemus unirersitatem, preter Psitucos sine dubio Primutes, prater Columbus quae Passeres cum Gullinis conjungunt, Trochilos, Picos, Coccyzespue etian segregarem libenter, majus quoties lucrum in scientiam redundaret. Quid cnim intererit, quicso, familias hasce, utpote notabiliores, in ordines elevare, cateris in acervum quoddam indigestum manentibus? 
1. Psitaci (Prehensores). Digiti bini antici, binique postici : tarsi reticulati: rostrum aduncum, cerigerum ad basim.

2. Accurreses (Rapaces). Digiti tres antici, unus posticus: rostrum aduncun, cerigerum ad basim: nares patule: ungues retractiles.

3. Passeres (Folucres). Digiti, vel tres antici unusque posticus, vel bini antici, binique postici : tarsi sentati : rostrum nec aduncum nec cerigerum.

4. Columbe (Giratores). Digiti tres antici, unus posticus: rostrum fornicatum, ceromate molli tunescente ad basim.

\section{Subclassis 2. GRALLATORES.}

Digitus posticus (pollex) altius tarso insertus quam antici, parum vel nihil solo insistens, minime constrictor, aut nullus. Tectrices atarum magne. Polygame plere: pullorum alacrum ac per se victituntium erlucutrices.

5. Gallinæ (Rasores). Tarsi teretes, ralidi, breviculi : tibiae totae plumosie. una cum apice femoris exscrtx: lostrum breve, fornicatum. Plumue compacter.

6. Struthiones (Ratite). Tarsi teletes, validi, longi : tibia seuinudae, una cum apice femoris exsertie: rostrum vel breve vel elongatum : sternum ecarinatun! alae impennes! Plume lacerte, laxissimu.

7. Gralla (Cursores). 'Tarsi teretes, tennes, elongati : tibia fere semper seminudre, una cum apice femoris cxsertæ: rostrum utplurimum elongatum. Plimuce compacte.

8. Anseres (Natatores). Tarsi compressi, breves: tibia fere semper seminudæe, earmmque bases una cum toto femore inserta: pedes palmati. Pluma densissima, compacte, oleose, lamigine ad basim circumsepte.

\section{Conspectus Famliarum et Subfaniliarum.}

Subchassis 1. INSESSORES.

Ordo 1. PSITTACI.

1. Psitcacid c. Lingua crassa, camosa : digiti non versatiles; antici distincti : tectrices alarum magnæ. 
1. Hacrocercima. Rostrum maxilla valde uncinata, mandibula valde profundior quam lata: cauda longissima, cuneata.

2. Psittacina. Rostrum maxilla conspicue dentata, mandibula ninus profunda quam longa: canda brevis, vel truncata, vel rotundata.

3. Plyctolophima. Rostrnm perbreve, latissinum, culnine mirifice incurvum : caput magnum crista explicalbili : cauda elongata, lata, rotundata, rectricibus largiculis.

4. Lorime. Rostrum leviter incurvmm; maxilla margine sinuata, vix enargrinata; mandibula gracili, conica, valde longiore quam profunda, gonyle recta.

5. Pezoporina. Rostrum validum, solidum, dentatum, culmine valde convexo; mandibula brevissima, profunda, gouyde curva: tarsus exilis, digito postico longior; digiti graciles: cauda longa latissima, valde cuneata.

Ordo 2. ACCIPITRES.

2. Vilturude. Caput subnudum, phumulis raris obsitum : ocnli litterales: talsus digito medio brevior: pollex subeleratus: ungues parum curvi. vix retractiles: alic magnae.

6. I ulturina. Rostrum minime dentatum, cera ylabra.

7. Gypactina. Rostrum elongatum, minime dentatum, cera setis tecta.

3. Grpogfrinide. Caput phumis densis tectum: oculi laterales: tarsus plus duplo longion digitis anticis.

8. Gypogermima. Rostrum loreve, classum, ricotu amplo: ale spina almatx.

4. Falconide. Caput plumis densis tectum: oculi laterales: cera patens: tarsus mediocris: pollex validus, insistens.

?. Polyborince. Rostrum brevienlum, sinuatmm : facies plus minus nuda! pedes tenues, tarsis digito medio vix longior' digiti laterales aequilongi : alie magnae, renigibns tertia et quarta omnium longissinis.

10. Aquilime. Rostrum longieulum, recticulum arl basim, simuatum: pedes validissimi: mngues robusti, valde adunci: alae magne, elongatre. lemigibus primis gradatis, guarta omnium longissima.

11. Buteonimer. Rostrum mediocre, crassmm ad basim, vix sinuatum: cera 
valde protensa : pedes modici, pollice brevi : alæ elongatæ, remigum quarta omnium longissina: cauda morlica.

12. Milince. Rostrum parvulum, crassum ad basim, sinuatum : cera protensa: pedes breves, pollice breviculo: alæ longissimæ, remigum quarta vel tertia vel secunda omnium longissima: cauda elongata.

13. Falconince. Rostrum breve, a basi abrupte incurvum, apiee utrinque dentatum: cera brevis : nares rotundre, pistillatæ: pedes mediocres, tarso tenui, nudo, digito medio longissimo, pollice brevissimo: ale elongatæ, acutæ, remigum secunda omnium longissina.

14. Accipitrince. Rostrum breve, a basi abrupte incurvum, sinuatum: pedes modici, tarso nudo, digito medio longissimo, postico parum breviore quam interior: unguis internus externo subduplus : alæe breves, remigum quarta omnium longissima.

15. Circince. Rostrun parvulum, rictu amplo, compressum, ad basim profundum, vix sinuatum : cera ad medium protensa : pedes elongati, graciles, nudi: alæ elongatæe, remigun tertia sen quarta omminn longissima.

5. Strigide. Caput ingens plumis densis tectum : oculi magni, faciales : cera obsoleta: rostrum breve, compressum : pedes plumulosi, digitis fissis, externo versatili.

16. Surnince. Caput modicum : discus facialis, intraocularis tantum, obsoletus: aures parvulie, inopereulatæ.

17. Bubonince. Caput grandiculum: discus facialis, intraocularis tantum, imperfectus : anres mediocres, inoperculatr.

18. Ululince. Capat grande: discus fucialis, oculos excedens, perfectus: aures grandes, operculatre.

19. Strigince. Caput immane: rostrum elongatun, ad basim recticulum: discus facialis, oculos excedens, maximus, excultissinns: aures ingentes, late operculatæ.

Ordo 3. PASSERES.

* Tribus I. Ambulatores. Digiti tres antici, unus posticus.

6. Caprinulgide. Rostrmm brevissimum, depressum, ad basim dilatatum, rictu immani: pedes brevissimi, graciles; digiti antici basi direl- 
gentes, membrana juncti; pollice versatili; unguis medius intus oblique dilatatus: ala magnæ rotmdate, pennis cubiti magnis: pluma omnes laxic, mollissinie.

20. Podargine?. Rostrum grandiculum, validiculum : unguis uredius margine integer : pollex normaliter situs.

21. Caprimulgina. Rostrum exillimum, lateribus inflexun : tarsus brevis; pollex contraversus; digitus exterior et interior aequilongi : unguis medius pectinatus.

22. Scorthormithine. Rostrum exillimum, lateribus inflexum : tarsus brevis; pollex contraversus: digitus exterior interiore brevior: unguis medius pectinatus.

7. Cyselma. Rostrun brevissimum depressum, triangulare, tcume, dilatatum ad basim, gonyde adscendente, marginibus infexis, rictu amplissimo: pedes exiles, brevissini, digitis anticis subequilongis: alse perlongat, falcate, tectricibus mediocribus pennas cubiti brerissinas abscondentibns: plume ommes strictie, nitentes.

23. C'ypselinre. Digiti onnino hissi : pollex debilis, rersatilis: nngues magni, valde curvati.

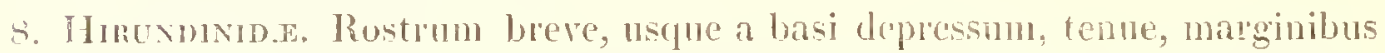
umbime inflexis, rictn amplo, glabro, vibrissis mullis: pedes breves: digiti subliberi, laterales requilongi : medius tarso longior: alde clongatae, tectricibus parvis, pennis enbiti longiculis: plumie omnes strivite, nitentes.

24. Irimmdiminre. Pollex breviol quam digitus internus: ungais unus nedins, areic interna integerrina.

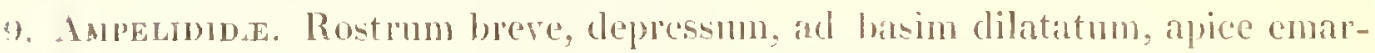
ginatum, ileflexmm; sutura incuata, guathidijs humilibus rerticalilus, carina rotundata; rictus amplissimus : ala, remigibus primaris decem, pedesque mediocres: plantae planat.

2.). Ampelidiure. Rostrun exile, gongle hand adscendente: rictus laxis: nares goundes, membramula postice clause: digiti laterales inatoules. exteriore: cum mediano conjuncto: tarsus squamulis lateralibus numerosis, reticulatis: alae modice elongatce, amplic, remige prima bro.. viore quam secunda. 
26. Bombycillince. Rostrum breve, durum, latum, subteres quasi triangulare, mandibula validicula, gonyde adscendente: rictus lævis: nares rotundatæe absque membrana, plumularum vellere semiclausæ: pedes validiculi, breves; digiti laterales subæquilongi: alæ elongata, acutre, remige prima longioribus aequali.

27. Gymoderince. Rostrum sub oculos usque fissum; nares absque membrana: facies jugulumque subnuda: plume capitis breves vel nulla: tarsi scutis fere novem, prominulis, subæqualibus : digiti subliberi, laterates inequales; pollex brevior quam digitus internus.

28. Queruline. Rostrum validum, latum, valde depressum; rictu setis rigidis instructo: digiti laterales inaquales, exteriore cum mediano conjuncto: tarsus squamulis lateralibus minutis.

29. Enryluminee. Rostrum breve, rectum, enormiter latissimum; maxilla valde convexa, dilatata ad basin, marginibus mandibulam amplectentibus, apice abrupte inflexo: pedes validiculi : digito externo cum mediano semiconjuncto; postico longiculo, interno omnibus breviore: alke brevicula: cauda brevis.

10. Corachadide. Rostrum breviculum, rectum, lateribus valde compressis, dilatatum, apice incurvo: rictus amplissimus, barbatus: nares lineares: pedes breves, digitis sejunctis, anticis ad basim parallelis, postico valde breviore.

30. Coraciadine. Lingua apice fimbriata: alae mediocres.

11. Priontrinæ. Rostrum mandibula utraque subincurva, compressum, marginibus denticulatis: digiti antici ultra medinm concreti.

31. Prionitince. Lingua longa, gracilis, margine ciliata: alæe breves, rotunditi: canda longa, cuneata.

12. Alcedride. Rostrum subquadrangulari-acuminatum, rectum; rictu amplo: pedes parvuli, digitis externis ultral medium concretis: tibiæ seminudre: alre breves, rotundatæ.

32. Dacelinine. Rostrum cylindraceum, ad basim depressum, lateribus dilatatum, gonyde adscendente: lingua brevis, apice triangulari.

33. Alcedimince. Rostrum a basi compressum, apice acutum, sutura rectissima: lingua brevis, apiee triangulari.

13. Meropid.e. Rostrum subquadrangulari-acuminatum, subcurvatum; rictu YOL. XVIII. $2 \mathrm{~N}$ 
amplo: tibix seminuda: pedes minuti; digitis externis ultra nedium concretis : alae elongatæ, acutæe.

34. Meropince. Lingua angusta, apice lacero.

14. Upupde. Rostrum elongatum, gracile, curvatum, valde compressum, intus parum exeavatum; marginibus integris oppositis; sutura elongata, recta: lingua brevissima, triangularis, obtusa, integerrima: digiái grandiculi, crassiculi, internus multo brevior, exteruns cum mediano semiconjunctus : ungues parvi, minime incurvi : alæe amplæe.

35. Upupine'. Maxillae intus perfecte plane: unguis posticus recticulus: cauda brevis, truncata.

36. Promeropince. Maxille intus aliquantulum concave, marginibus acutis: unguis posticus curvus : cauda longa, cuncata.

15. Paradjsejde. Rostrum grandiculum, cultratum, subemarginatum, sub oculos usque fissum: capistrum plumulis holosericeis densis nares occultantibus: pecles maximi, cute molli, vix scutati tccti; digriti tennes, elongati, internus multo brevior, a basi divergens, extermus articulo sesquialtero concretus; pollex maximus.

37. Paradiseimre. Lingua acuta, apice laciniata: alæ longre, amplie: penna hypochomlriace elongatissimæe.

16. Melliphagm.e. Rostrum longum, modice gracile, emarginatum: pedes valicliculi, digito postico clongato.

38. Melliphagine. Lingua longe extensilis, membranacca, apice penicillato.

17. Crnninde. Rostrun longum, graeile, phus minus curvum, integrum: nares breves, ovales, membranacea, rima laterali apertie: pedes modici: ala mediocres, remigibus primis plus minus gradatim abbreviatis.

39. Cerebince. Rostrum tenue, subcompressum : lingua longe extensilis. longe ciliata.

40. Cymmirine. Rostrum longum, tenue, minime compressum: lingui Ionge extensilis, membranacea, glabra.

18. Trochunde. Rostrum Iongum, gracillimum; rictu minimo: lingua vibratilis, tubulosa, suctoria: pedes exigui : alie longissima, falcate.

41. Trochiline. Rostrun tereticulum, tomiis maxillaribus mandibularia 
amplectentibus, myxa brevissima: digiti basi connati, subtus plani : ungues parvuli.

19. Certhide. Rostrum longulum, compressum, integrum : lingua elongata, non extensilis, apice cartilagineo: pedes brevicnli; digiti clongati, validi, compressi: pollex validissimus; ungues grandes, arcnati, acuti : cauda cuneati.

42. Tichodromince. Rostrum integerrimum; membrana narium muda, fornicata: tarsus digito postico breviculo longior; digiti laterales subæquilongi a basi disjuncti : canda mollis, brevicula.

43. Thryothorince. Rostrum subemarginatum, membrana nariun fornicata: digiti antici ad basim adnati: canda rotundata, vel gradata, brevicula, mollis.

44. Certhine. Rostrum plus minus curvum, valde compressum; lingua acuminata: digiti antici ad basim conjuncti: rectrices rigidae, rachide excedente.

45. Anabatidina. Rostrum breviculum, validum, rectum, subemarginatum ; membranas narium fornicata: digiti modici, fere disjuncti, medius longior quan laterales: remiges breves: canda cuneata, rectricibus, rachide non excedente, rigidis.

46. Sittince. Rostrum rectum, seu adscendens, conicum, acutum; membrana narium plumata sen tecta; lingua lacera: digitus posticus medio xquilongus: ala longæ, acutre, remige prima vix secmnda breviore: canda mollis, brevissima.

20. Paride. Rostrum breve, conico-subulatum, durissimum, integerrinum; mandibula marginibus simplicibus, hand inflexis: nares plumulis densis, setaceis, tectie, membrana obsoleta: pedes validi; digiti subfissi, internus omnium brevissimus: ungues validi, valde incurvi; posticus maximus.

47. Parince. Alæe breviculæ, rotundatæ, remigibus tribus prinis gradatis.

21. Tanagride. Rostrum breviculum, crassiculum, conico-trigonum, maxillis æqualibus, superior apice utrinque emarginato, subdeftexo, inferior marginibus simplicibus hand inflexis: nares nudxe, membrana fere obsoleta, carina interjeeta: vibrisse parve, adpressi: pedes mediocres. 
48. Piprince. Rostrum brevissimum, sub oculos usque fere fissum ; maxilla valde incurva : pudes elongati, grateiles; digitus externus longior quam internus, articulis tribus cum mediano coneretus: ala mediocres: cauda brevis, rectrieibus duobus mediis quandoque elongatis.

49. Tamagrince. Rostrom mediocre, validum, nodice fissmm; maxilla partun incurva: pedes breves; digiti subliberi; pollex digitique laterales aquilongi, articulum secundum digiti medii vix supelantes: ungues lati, incurvi: alae candarne longiculie.

22. Alatdida. Rostrum conico-attenuatum, minime emalginatum, mandibula marginibus simplicibus, haud inflexis: nares membrana fornicata nudie: pedes mediocres, digitis tenuibus, subliberis: ungues vix curvati, postieus digito praclongior.

50. Alandince. Alx remigibus tertiariis elongatis, secundariis apice enarginatis.

23. Motacilude. Rostrum longrenlum, rectum, subulatum, ntringue cmargrinatum; mandibula marginibus simplicibus, hand inflexis: pedes longiculi, digitis tenuibus, exteriore cun mediano basi concreto. postico omminu valde lomgiore.

51. Motacillince. Ale acuminate renigibus tertiariis elongatis, sceundauris apice emarginatis : cauda prodncta, angusta.

24. 'T'ervide. Rostrum subcultratum, ufrinque chnarginatum, mandibula malginibus simplieibus, luand inflexis: nares nude, membrana cartilaginea fornicata : podes digitis lateralibus subliberis, inerinalibus, interno vix excedente articulun secundum medii : ungues ineojuales: penna cubiti aerualles.

52. Cinclince. Rostrun mediocre, rectun, clepressiculum, vix emarginatum, gonyde adscentente, marginitus contratis: nates lineares: pedes robusticuli; digiti laterales apduilongi; squante tarsi integræ.

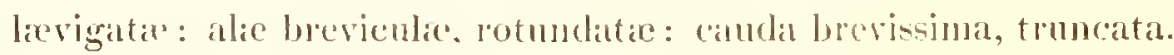

53. Myiotherime. Rostrum rectum, subcylindraceum, apjec abrupte inHexo: pedes clongati; digiti laterales subcenilongi, internus usque a basi separatus, divergens : ala breves, rotmudate, lornicatæe, remigibus brevissimis tectis: cauda brevis.

54. Ixodina. Rostrum distincte emanginatum: pedes brevissimi, digito 
postico tarso subrequilongo: ungues breves, lati, valde incurvi : alae breves, rotundatre: uropygii plumae elongata, confertissima.

55. Turdince. Rostrum emarginatum, culmine gradatim incurvo, sutura curvata : pedes mediocres: alæe longiculæe, acuticula.

56. Timalince. Rostrum rectum, compressum, durum, vix emarginatum : pedes grandes, validi; digitus internus usque a basi separatus, divergens: ungues parum incurvi: alae breves, rotundate, remigibus brevissimis, tectis: cauda mana, lata, gradata, mollissima: pluma ommes laxæ.

57. Oriolince. Rostrum longitudine capitis, latum ad basin, validum, compressum; rictus ampliculus; vibrissis nullis: pedes breves; digiti laterales inaequales: alae elongatae: plume nopygii confertae.

58. Leiothricince. Rostrun lobustmu, gonyde adscendente: pedes grandes, robusti; digiti externi ad medium usque conjuncti, posticus exteriore longior: alæe breves, rotundatre.

59. Fireomince. Rostrum robusticnlum, compressum; maxilla utrinque emarginata, apice inflexo; mandibula apice adscendente: vibrissa rigidula nares tegentes: pedes robusticuli; digito interno omnium brevissimo: alae longicula, subacutre: cauda mediocris, vel truncalta vel emarginato-rotundata.

60. Calamoherpince. Rostrun subulatum, usque a basi compressum, emarginatum; nrandibula tenuior maxilla: pedes validiculi, elongati : ala breviculae, subrotundatæe, remigum spuria minima extante, prima et secunda omnium longissima.

61. Sylence. Rostrum gracillimum, subulatum, usque a basi compressum, emarginatum; mandibula tenuior maxilla : pedes graciles, longiculi: alæe longiculæ, acuticula, remigum spuria laticula extante, secunda et tertia omninm longissince.

62. Saxicolince. Rostrmon ad basim depressum, sntura recta; vibrissis divergentibus: caput grandiculum : pedes longiculi : ala elongata, aupliculae: cauda brevicula, laticula.

63. Sylvicolinee. Rostrum compressum, trigono-subulatum, vix emarginatum: pedes graciles; digiti laterales inrequales, pollex validiculus: alce longicula, acuticula, remigun spuria nulla, tribus primis subxqualibus ominim longissimis. 
25. Muscicapide. Rostrum usque a basi valde depressum, latum, maxilla marginibus mandibulam subtus late convexam amplectens, apice emarginato abrupte inflexa; rictus ampliculus, vibrissis porrectis: menbrana narium obsoleta vel nulla: pedes breviculi, tenuiculi; digiti laterales articulos duos medii excedentes: ungues inaequales parum curvati.

64. Muscicapima'. Rostrum mediocre, rectum, plus minus depressum, medio carinatum; rictus vibrissis rigidis elongatis: pedes breves, gracillimi: digiti laterates insequales, exterior cum mediano conjunctus: alae longicule : cauda elongata.

65. Treniopterince. Rostrum longiculum, parum depressum, rotundiculum, lateribus rectis; gnathidis verticaliter positis: pedes elongati, validiculi: ala remige prima modice elongata: canda longicula.

66. Tyramince. Rostrum usque a basi depressum; gnathidis subhorizontaliter positis; rictus vibrissis nares obtegentibus: pedes breves, graciliculi; digiti laterales subrequilongi : ungues elongati, graciles, incurvi, acutissini.

67. Edolinre. Rostrum ultra medium compressum, culmine sensin curvato: perles breves: alce elongatx, plus minus acute: cauda elongata, sapius forficata.

68. Ceblepyrine. Rostrum ad basim latum, gonthidiis subhorizontalibus, angulo frontali acuto, sutura arcuata, vibrissis vix ullis: nares rotundatre absque membiana, plunulis tectre: pedes breves; digiti laterales inaequales : ale elongatre, remigibus tribus primis gradatis: caucla medio emarginata; hine inde rotundata: pluma uropygii densissima, fere spinosie.

26. L.unida. Rostrum validiculum, conrexum, compressum, apice deflexo utrinque, vel cum dente exserto, vel pofunde emarginato, grnathidiis altis, verticalibus; sutura recta; ribrisse, nares rotundatxe absque membrama : pedes mediocres; pollex graciliculus; mgues acuti: ala mediocres, renige tertia seu quarti longiore.

69. Psaridince. Rostrum grandiculum, carssum, subcylindraceum, sinu rotundo frontem late intrans, apice ablupte inflexo ennarginatum; nares rotundata, nuda, parve: pedes exiles; digiti laterales in- 
æquales; squamæ tarsi anteriores transversæ, laterales parvæ, nиmerosæ: alæ longx.

70. Tamophilince. Rostrum elongatum, strictum; sutura recta, apice abrupte inflexo, dente utrinque acuto : digiti laterales iniequales; exterior et medianus articulo primo conjuncti : ungues lati, obtusiculi : alæe breves, rotundatie, fornicate, remigibus primariis decem pennas cubiti vix superantibus, brevissimis: canda mediocris, rotundata.

71. Lanince. Rostrum breviculum, dente utrinque acuto: digiti laterales æquilongi, liberi: ungues graciles, acuti : alæ nediocres.

27. Corvine. Rostrum robustum, vel conico-acuminatum, vel cultratum, frontem intrans cum basi : nares vel plunis setaceis recumbentibus, vel vibrissis tectie: pedes validi.

72. Garruline. Rostrun apice leviter deflexo, utrinque emarginato: nares rotundatre absque membrana: pedes digitis lateralibus inæqualibus: alæ breviculæ, subrotundæ: formæ elegantes: plumæ nitide coloratæ.

73. Corvine. Rostrum ad basim cute crassa obvolutum, vix emarginatum; nares plumulis setiformibus densis contcetx, sutura recta; gnathidia valida, linearia, recta: pedes robusti, scutis prominulis: digiti subliberi, laterales subæquilongi : alæ longæ, acutæ: formæ graves: plumæ plus minus atratæ.

74. Baritince. Rostrum longitudine capitis, robustum, rectum, conicocompressum, apice parum subadunco, utrinque profunde emarginato, ad basim superne latum, circulariter frontem intrans; nares parvæ, nudæ, lineares, absque membrana; sutura recta : pedes magni, scutis lævibus; digiti subliberi, lateralibus subæquilongis, pollice maximo : alæ elongatæ.

75. Glaucopince. Rostrum breve, culmine elato, a basi gradatim curvatum, integerrimum; mandibula apice recto; sutura valde curva; rictus lævis: alie breves, rotundæ: cauda elongata, gradata.

76. Coracinince. Rostrum robustum, prædurmm, rectum, compressum, ad basim depressum, culmine subcurvato tantum ad apicem, vix emarginatum; mandibula apice recto, depresso; rictus vibrissis raris: nares semiplumulosæ: pedes breves, validi; digitis anticis subæqualibus: alæ elongatæ: cauda brevis. 
77. Sturnina. Rostrum longulum, conico-acuninatum, integrum, utrinque angulatum ad basim, apice depressicnlo, obtusicnlo, subdeflexo; sutura ad basim deffexa; gnathidia alta, verticalia: nares nulæ: frons convexa: pedes scutati, digitis subliberis, lateralibus æequilongis, interno divergente, pollice maximo: remiges primariæ decem: plumix capitis elongatæ, angustæ.

78. Lamprotornithine. Rostrum breviculum, compressum, utrinque angulatum ad hasim, cutuine a basi curvato, integrum: pedes digitis lateralibus inæerqualibus: rcmiges primarix novem: plumie capitis elongatie, angustie.

79. Quiscaline. Rostrum valiulum, a fronte recte depromptum, conicodongatissimum, compressum, ntrinque ad basim angulatum, culmine leviter curvato: pedes robusti: remiges primarixe noveur: cauda graditti, navicularis: plumæe eapitis rotundatid.

80. Icterime. Rostrum a froute recte depromptum, breviculum, conicum, ntrinque ad basim angulitum, integrum ant rectum, ant mandibulis binis gradatiun subincurvis: pedes validiculi, digitis lateralibus æquilongis: ungues classi, vahle curvati: reniges primaria novem: plume capitis rotumdate.

228. Fraxilume. Rostrum breve, validum, conicum, crassissimum ad basin, mandibulit nurginibus validis curratim intra se convergentibus, postice altioribus: pedes graciles.

sl. Ploceine. Rostrum rectum, conicum, culnine depresso saltem posterius, angulo basilari lato, triangulari, frontem valde intrante: nares plano intervallo distantes: remiges primarie decem, prima minuta.

82. Emberisine. Rostrun conicum, culmine recto, marginibus intractis, maxilla angustiore (fuan uaulibula; tubereulo osseo longitudinali ad palitum : wares plano intervallo distantes: pedes mediocres; ungues graciles, incurvi: remiges mimarixe novem.

83. Fringilline. Rostrum plus nuinus robustum, perfecte conicum, culmine tereti, saltem posterius; angulo frontali brevi, acuto: mandibulis serualibus, subincurvis ad apicen tantum, integris: nares plano interallo distantes: remiges primarie novem, prima deficiente.

84. Loxintr. Rostrum brevissimum, crassum, integrum, supra infraque curvatum; maxilla longiore, culunine marginibusque incurvis: nares 
plano intervallo distantes: alæ remigibus primariis novem, primis quatuor snbæqualibus.

85. Pytilince. Rostrum breve, crassum, subtrigonum, utrinque subemarginatuin: angulus frontalis angustus, acutus, valde intrans: nares approximatie carina tantum interjecta: alæe remigibus primariis novem, primis quatuor subequalibus.

86. Phytotomince. Rostrum breve, marginibus serratis; inaxilla profunda, culmine arcuato; mandibula tenuis: nares plano intervallo distantes: pedes breves, validi, digitis duobus vel tribus anticis, uno postico: remiges primarix novem.

29. Colide. Rostrum brere, integrum; maxilla ad basim dilatata, convexissima, culmine elevato, arcuato: pedes robusti; digiti fissi, ommes antrorsum versi :

87. Colince. Nares nudæ: alæ breves, acutæ: canda longa, euneata: plumæ mollissimæ.

30. Bucerontidx. Rostrum enorme, iname, variatim appendiculatum, valde compressum, mandibula utraque incurva, marginibus integris : pedes magni, digitis externis ultra medimn concretis.

88. Bucerontince. Digiti laterales inæequales; medius longitudine tarsi; pollex omnium brevissimus: ale breviculæ, rotundatæe: cauda longicula, rectricibus rotundatis, ad basim angustis.

\section{** Tribus II. Scansores. Digiti bini antici, binique postici.}

31. Ranphastidide. Rostrum immane, vacuum, marginibus serratis: digiti externi internis Jongiores, bini antici non ultra medium fissi: alæ breves, rotundate, tectricibus magnis.

89. Ramphastidince. Lingua penniformis: scuta tarsi sex: alre breves, rotundatie.

32. Picides. Rostrum rectum, polyedrum, apice cuneato: lingua lumbriciformis: digiti antici ad basim connati: tectrices alarun breves.

90. Picine. Rectrices rigidæ, acuminatæ.

91. Iungince. Rectrices molles, rotundatæ.

33. Buccoside. Rostrum rectum, conico-compressum, robustum, ad basiul crassum, setis elongatis circunsessum : pedes grandiculi : digiti exYOL. XVIII. 
terni internis longiores, antici toto articulo primo connati, exterior posticor'um versatilis, liber.

92. Buccomince. Alie breves: cauda brevis, mollis: ungues omnes inenrvi.

34. Cuculdis. Rostrum tenue, setis vix ullis; lingua plana: tarsi scutati: digiti antici fere fissi, exterior posticorum versatilis, liber.

93. Cuculine. Rostrum tenue, convexum; nares rotundre, margine prominente: pecles parvi : tarsus brevissimns, scutis quinque: digriti subtus molles, incrassati : alie acutce.

94. Coccyzince. Rostrum incurvum, marginibus maxilluribus dilatatis: nares lineares, simplices : pedes grancliculi ; tarsus elongatus, nuclus; digiti ad medimn teretes: alre breves, rotundatre: cauda longissina cuneata.

95. Crotophagince. Rostrum latum, compressum, culmine elevato: natres simplices: pedes grandiculi, digitis ad medium teretibus.

96. Saurotherince. Rostrum elongatum, culmine convexo: nares simplices: pedes grandieuli, digitis ad medium teretibus.

97. Indicatorince. Rostrum breve, subconicun: nares simplices: pedes brevissimi.

35. Captonide. Rostrum rectum, compressum, rictu amplo, setis elongatio circumsessum: pedes debiles.

98. Capitoninee. Alie rotundatie.

36. Galbulid Rostrun elongatmm, rectum, quadrangulare, integerrimmu: rictu amplo, vibrissis validis: pedes debiles; digiti externi internis longiores, bini antici ad apicem tantum discreti, interior posticorum brevissimus vel nullus.

99. Galbuline. Alie breves: cauda elongata, gradatá.

37. 'Trogovid e. Rostrum breve, validum, triangulare, convexum, apice utrinque enarginato, rietu amplo: digitus secundus simul eun pollice letroversus : tertius et quartus antrorsm versi, concleti ad basin. interni externis longriores.

100. Trogonine. Pedes parvi, semihisuti : alae brevissime.

3s. Musomagnde. Rostrum brere, compressiculum rems apicen, maxilla profunda, culnine arcuato, marginibus serrulatis, mandibula tenui : 
pedes breves, vix scansorii, digitis anticis tribus membrana connexis, externo subversatili, pollice omnium minimo: alæ breviculæ.

101. Mhsophagince. Cauda longa, rotundata, rectricibus latis decem.

\section{Ordo 4. COLUMBE.}

39. Colunidide. Rostrum breve, tenue, debile, fornicatumı; cuticula mollis, tumida, naribus imposita: pedes breves; digiti subtus molles, subincrassati; plumæ corporis densæe, rachide ad medium crassiore: plumæ uropygii rigidulæ.

102. Columbince. Digiti omnino fissi, parum divergentes: pedes parvuli, pollice insistente; tarsi scutati : alæ longiculæ.

103. Ptilophyrince. Digiti plica cutis ad basim juncti, valde divergentes: pedes validiculi, pollice subelevato; tarsi reticulati: ale breves: cauda longa.

\section{Subclassis 2. GRALLATORES.}

Ordo 5. GALLINA.

40. Pterochids. Rostrum tenue: alæ longæ, acutæ: cauda subelongata, acuta.

104. Syrhaptince. Digiti tres coadunati, solo apice distincti.

105. Pteroclince. Digiti quatuor, anterioribus membrana connexis ad basim.

41. Penelopide. Rostrum validum, durum : digiti a basi divergentes, membrana comnexi; ungues magni, compressi, acuti ; pollicis insistens major: alæ breves: cauda elongata.

106. Penelopince. Pedes elongati, crassi.

42. Phasmanide. Rostrum subvalidum, pre naribus excisum : digiti antici membrana connexi ad basim; pollex longiculus: alæ breves, rotundatæ: cauda producta.

107. Pavonince. Cauda in flabellum explicabilis, tectricibus productissimis.

108. Phasianince. Canda compressa, inexplicabilis.

43. Tetraonide. Rostrum validum, continuo convexum, durum: digiti an202 
tici membrana connexi al basim: ale breves, rotundata: cauda brevissima.

109. Perdicince. Niures nudie: tarsi implnmes: canda perbrevis.

110. Tetrumine. Nares phumulose: supercilia glabra: tarsi plumosi: cauda longiculi.

44. Cirpturida. Rostrum tenue: digiti fissi : alre breves, rotundatæe cauda aut perbrevis aut nulla.

111. Ortigydine. Rostrum compressum : digiti tres, pollice nullo.

112. Ciypturince. Rostrum depressum: digiti quatuor, pollice parvo.

Ordo 6. STRUTHIONES.

45. Struthonde. Digiti fissi duo tantum vel tres: tarsi seutulati : alie breves, abortive, remigibus nullis.

11:3. Struthionine. Rostrun breve, crassum, gallinacenm: pedes ecalcarati.

114. Apterygine. Rostrum elongatum, gracile, grablacenul pedes calcarati.

Ordo 7. GRALL.E.

16. Cularadridas. Rostrum mediocre, validiculum: pollex mullus ant brevissilmus.

15. Otidine. Rostrum breve, subfornicatum : pedes validi, reticnlati. digitis tribus, anticis, beribus, membranula connexis: mugues fornicati nee solidi: ala breves, amples, remige tertia omminm longrissima.

116. Charudrine. Rostrum breve, molle juxta basim, ipice incrassato: sulcus nasalis profundus, ad medium rostrum extensus: pedes invalidi; digiti tres antiei cylindracei; posticus fere semper nullum: alie longa, acute, remignm prima omnimm longissima.

117. Hematopodime. Rostrum rectum, robustum, valule compressum. acutun: pedes breviculi; digiti omnes membrana connexi; interjor omnium brevissimus: ale longx: canda brevis.

47. ('Hundomes. Rostrum breve, validum, compressum, jntegrum: pollex brevissimus, introversus.

11s. Chionidina. Rostrun tectum ad basim vagina cornea, antice libera: 
nares tubulares: pedes brevissimi, validi: squamæ tarsi parvulæe, scabre: digiti exteriores membranula ad basim connexi, internu fissus: ungues fere detriti.

119. Thynochorince. Rostrum crasse, conicum : digiti mediocres : alce acutie, falcatre.

48. Psophide. Rostrum breve, subfornicatum : digiti antici membrana conjuncti; pollex satis excultus.

120. Palamedeince. Pedes validi, crassi, reticulati; digiti longissinni : pollex robustus, fere totus insistens: ale ample, bicalcaratre.

121. Grume. Pedes longi, graciles, scutati; digiti mediocres; pollex minutus, vix solo apice insistens: alæe amplie, breves, concave.

49. Ardende. Rostıun longmu, validum: pollex longus, pluribus articulis solo incumbens: alie magnie.

122. Ciconince. Rostrum crassum, conico-elongatum, vix ante oculos fissum, rectissinum, culmine juxta medium depresso; maxilla lavi : caput plus minus implume: unguis medius integer: pollex a solo elevatus.

123. Ardeine. Rostrum longum, rectissimum, conico-elongatum, conpressum, sub ocnlos nsque fissum; maxilla ntrinque longe sulcata: unguis medius serratus.

124. Cancromince. Rostrum breve, enormiter latissinum, navicnlare: maxilla inanis secantes mandibule margines operiens.

125. Plataleince. Rostrum longum, planmm, apice valde dilatato, rotundato: pedes semipalmati.

50. Tantaljox. Rostrum prælongum, arcuatum: facies implumis: pollex longus, solo fere totus incumbens.

126. Tantolince. Rostrum apice obtuso rotundato, marginibus contractis secantibus: pedes elongati, digitis anticis membrana conjunctis.

51. Scolopacidas. Rostrum elongatum, graeile, molliculum; sulco nasali fere ad apicem rostri continnato; rictus minimus : pollex brevis, gracilis. vix insistens aut nullus: aloe longie, acute: canda brevis.

127. Tringince. Rostrum vel longissinum vel breviculum, apice laevigato, haud crassiculo: digitus medius tarso brevior; pollex brevissimus ant nullus. 
12S. Scolopacina. Rostrum longissimum, rectum, apice l'uguloso-scabro, in maxilla longiore subtus crassiculo: digitus medius tarso valde longior : pollex satis excultus.

52. Rallide, Rostrum breve, vel mediocle, crassiculum, compressum: pedes magni, classi; digitis fissis longissimis; pollex longulus articulo primo insistens: alic breves, rotundate: canda brevissima, a tectricibus abscondita. Cormus compressissimum: habitus gallinamu.

129. Parince. Rostrum rectum : pedes longissimi, digitis gracilibus et ungubus praelongis: ala siepius spinosa.

130. Rallinre. Rostrum subcurvatum: pedes mediocres, digitis crassiculis, ungubus congruis: alce inermes.

53. Phalaropodnde. Rostrum mediocre, rectnm, gracile, flexile: pedes breves; tarsi compressi ; digiti lobati.

131. Phaluropodince. Maxilla utrinque sulcata, apice deflexo, acnto.

5. Recurvinostride. Rostrum rectum, ant etiam recurvmu, tenuissimm, flesile: perles longissimi, debiles; tarsi compressiculi ; digiti semipalnati.

132. Recurvirostriuce. Rostrum teres, acuminatum.

55. PHENICOPTERIDA. Rostrum hreve, crassum, mellio infuctum, marginibus limelloso-denticulatis: pedes longissimi, palmati.

133. Phonicoptevine. Maxilla intus solida, cirinata, depressissima, valde cxilior quam nuandibula.

\section{Ordo S. ANSERES.}

56. Axatude. Pollex palrus, liber: rostrum cute molli tectum, ungulatum ad ipjicen, depressum, marginibus lamelloso-denticulatis: lingua crassa, carnosa, lateribus dentatis.

134. Cyguince. Rostrum basi tunnidum, cornemm, ad oculos usque implume, denticulis lanellosis compressis : collum longissimum: pedes breves, lati; pollex simplex.

135. Anserince. Rostrum breviculum, ad basin profundum, cornemm, plumosum, denticulis abbreviatis, conicis, acutis: nares ad medium rostri: perles longiculi, fere equilibres: pollex simplex.

136. Auatine. Rostrum longiculum, latum, denticulis lamellosis elon- 
gatis, compressis: collum breviculum: pedes brevissimi : pollex simplex.

137. Fuliguline. Rostrum mediocre, latum, denticulis lamellosis, clongatis, compressis : nares ad basim rostri : collum brevicnlum : pedes exeentrici; pollex dilatatissimus, lobatus.

138. Mergine. Rostrum elongatum, angustum, apice abrupte inflexo, rotundato, marginibus acute serratis: pedes breves, postice excentrici; pollex dilatatissimus, lubatus.

57. Pelecanide. Pollex elongatus, subirsistens, membrana junetus (digitis quatuor, omnibus membrana lata connexis), vel lobatus : pedes brevissimi : alæ magnæ, valde elongatæ. In arboribus considunt.

139. Pelecanince, Maxilla crementaria.

140. Plotine. Mandibula utraque solida.

58. Liride. Pollex liber, elevatus, vel nullus: pedes requilibres: rostrum marginibus haud denticulatis; nares non protuberantes: alie elongatæ, acutæ. Bene ambulant: eximie volant: male urinantur.

141. Rhinchopince. Rostrum mirifice compressum, maxilla valde brevior quam mandibuli.

142. Sternince. Rostrum longum, compressum, apice recto acuninato: pedes breves: alie valde elongatæ: cauda longissima, forficata.

143. Larince. Rostrum mediocre, compressum, apice valde íncurvo; mandibula subtus conspicue angulata: pedes modici ; tarsus digito medio sublongior; pollex brevissimus: alæ longissimæ: canda brevicula, subtruncata.

144. Lestrince. Rostrum mediocre, ad basim rectum, cylindraceum, cer"a tectum, apice uncinato; maxillis cæmentariis; mandibula subtus angulata: pedes graciles; ungnes validi, curvati: cauda gradati. rectricibus duabus mediis productis.

59. Procellaride, Pollex vel nullus, vel tantum unguis; pedes excentrici: rostrum marginibus hand denticulatis: nares tubulares : alæe elongate, acutæ. Ire nesciunt: eximie volant: male urinantur.

145. Procellarince. Rostrum rectum, apice abrupte uncinatum, maxillis ciementariis.

60. Colmabide. Pollex parvus, liber: rostrum conico-subulatum, margini- 
bus haud denticulatis: collum elongatum: tarsi compressissimi: alæ breves, minutæ, falcatæ, subacutre. Lacustres ef murince. Incessus difficillimus, corpore erecto: volatus rectus, celer: urinatrices eximice.

146. Podicipinae. Rostrum snbconicum : pedes lobati: canda nulla.

147. Colymbine. Rostrum longulum, rectum, acutum: perles palmati: cauda brevis.

61. Accid.E. Pollex nullus: rostrum conpressum, culmine plus minus arenato, marginibus hand denticulatis : eollum breve: tarsi parum compressi: alæ breves, minutæ, falcatæ, subacutæ. Maritime. Boreules. Incessus difficillimus, corpore crecto: colutus rectus, celer: urinutrices eximice.

148. Phaleridime. Nares nudle: pedes palmati.

149. Alcince. Nares plumulis ohtectie: pedes palmati.

62. Sphenscus. Pollex grandiculns, liber, anteversus: cauda remigesque mullie.

150. Sphoniscince. Rostrum longum, tenue, mandibula sub basi incrassata: plumx omnes squamiformes: ale pimniformes. Oceano Antrerctico proprice.

\section{Chaseis III. AMPHIBIA.}

Animalia vertelsata, sangune frigido, circulatione duplici, imperfecta, ovipara aut orovivipara: pulmones bini vel unus, liberi: cor biloculare vel mileculure, biauritum: dentes fere in omnibus: corpus vel catapluactum, vel - guamosum. vel nudum.

\section{Ordixum Distributho.}

Subelassis 1. MONOPNOA (Ablopmon).

Respiratio ope julmonum tantum: metamorphosis nulla: corpus plus minus restitum: rondylus occipitalis simplex: penis: copulatio insita: ova crustacca aut coriacea. 


\section{Sectio 1. RHIZODONTA (Loricata).}

Dentes infixi (maxillarum alveolis injuncti): labia libera nulla: lingua adnata : os tympanicum cum cranio concretum : coste distincte : artus quatuor: penis simplex : an us longitudinalis.

1. Orvithosauri (Giyphi). Pedes tetradactyli ; antici digito quarto enormiter elongato (membranam alarem expansam ad sustinendam idoneo). Fossiles. Aerei.

2. Euydosauri (Crocodili). Pedes digitati, antici pentadactyli, postici tetradactyli, palmati vel semipalmati. Fluviatiles.

3. Enamosauri (Cetosami). Pedes breves, pinniformes (permultis ossiculis conflati ut in Cete). Fossiles. Marini.

\section{Sectio 2. TESTUDINATA.}

Corpus clausum in theca bivalvi, supra a costis concretis constituta, infra a sterno: os tympani cum cranio connatum : dentes nulli: lingua adnata: penis simplex: artus quatuor.

4. Chelonir (Testudines). Corpus reversum, testeun.

\section{Sectio 3. REP'TILIA (Squamata).}

Corpus squamosum : costa distincte, truncum fere totım complectentes : cranium suturatum: dentes in maxillis non inscrti : lingua libera: labia adpressa, margine libcra: penis duplex : anus transversus.

5. Sauri (Lacerter). Rictus haud dilatabilis: mandibula rami ad apicem per symphysim juncti : os tympani mobile: ossa faciei concreta, immobilia: oculi patentes: artus quatuor quandoque abortivi : sternum breve: clavicula: pulmones duo.

6. Ophid (Serpentes). Rietus dilatabilis: mandibula rami ad apicen ligamentis connexi : os tympani saltem mobile: oculi patentes: pedes, claviculae, sternum, pelvis, tertia palpebra, tympanum nulli: pulmo altel* abortivus vel nullus: lingua angustissima, bipartita, vibratilis, basi vaginata: corpus prælongum, teres.

7. Saurophidr (Angues). Rictus haud dilatabilis: mandibula rami and apicem per symphysim juncti: os tympani cum cranio connatum, oblique vOL. XVIII. 
promum : oculi parvi, sub cute latentes : tympanum mullum : corpus squamarmu rudimentis ammulatin caratum : artus plerumque vel duo vel nulli : pulmo mnicus, altero abortivo: lingua lanceolata, depressa, bifida, non vaginatil.

\section{Subclassis 2. DIPNOA (Diplopmoa).}

Respiratio ope pulmonum simulque branchiarum in prima saltem vitæ periodo: metamorphosis in pleris : corpus, vix paucissimis exceptis, mudum : condylus occipitalis duplex : penis nullus: copulatio vel ex contactu tantum vel nulla : ova membranacea.

\section{Sectio 4. BA'TRACHIA $\left(\boldsymbol{N}^{\top} u d \alpha\right)$.}

Coste imperfectr: lingua carnosa, adnata.

8. Batrachophon (Crecilier). Metamorphosi vix obnoxia: branchice evanidae: os tympani cum cranio commatum: corpus apodum, ceaudistum : anus teminalis, rotundus.

9. Rıxe (Batrachia erea). Metamorphosi obnoxia: branchice (in larvis tantum operculatie) deciduse: pedes quatuor.

10. Iснтнгорi (IChthyoidea). Metamorphosi non obnoxia: branchia persistentes: anus longitudinalis: pedes quatuor vel duo.

\section{Conspectus Familarum at Subfamliarum.}

Subclassis 1. MONOPNOA.

Sectio 1. RHIZODON'TA.

Ordo l. ORNITHOSAURI.

1. PTeronactrade. Caput lostratum: collum clongatum vertebris septem longissimis: costie tenues, elongatie, simplices.

1. Pteroductylina. Dentes aequales: orbitae maxima: caudi brevissima.

$$
\text { Ordo 2. EMIYOSAURI. }
$$

2. Crocodulade. Corpus loricatum; dorso transversim fiasciato sentellis osseis durissimis: maxilla simosa. 
2. Crocodilina. Dentes conici, inæequales: aures valvula obserabiles: cauda corpore longior, validissima, compressa, cristata.

3. Teleosaurina. Retro-nares maximæ, eoden plano ac fossa orbitalis sitæ.

Ordo 3. ENALIOSAURI.

3. Plesiosauride. Dentes alveolis distinctis inserti : rertebre planiculie.

4. Plesiosaurina. Caput minimum, rostratum, mandibula postice elongata; dentes nuncrosi: collum longissimum, vertebris numerosissimis : cauda brevis: pedes longiculi.

4. Ichthrosauride. Dentes sulco communi inserti : vertebra biconcave.

5. Ichthyosaurina. Caput magnum, orbitis maximis, acute rostratum: dentes numerosissimi (30-35 utrinque in utraque maxilla), aquales: costre numerosa, longiculæ, subtus conniventes: cauda longicula: pedes brevissimi, antici majores.

\section{Sectio 2. 'TESTUDINA'TA.}

Ordo 4. CHELONII.

5. Chelonide. Pedes natatorii, compressi, longitudine inæequales, digitis indistinctis: labia nullı.

6. Chelonina. Thorax scutis corneis tectus.

7. Sphargidina. Thorax corio verrucoso indutus.

6. Trioxycidæ. Pedes ambulatorii, longitudine pares: thorax corio læri indutus: labia carnosa.

8. Tryonicina. Pedes plantigradi, digitis distinctis, palmatis: os corneum : collum versatile: pelvis immobilis.

7. Testudnide. Pedes ambulatorii, longitudine pares: thorax scutis corneis tectus : labia nulla.

9. Chelydina. Pedes plintigradi, digitis distinctis, palmatis: os coriaceum : collum versatile: pelvis immobilis.

10. Hydraspilina. Pedes plantigradi, digitis distinctis, palmatis : os corneum : collun versatile: pelvis immobilis.

11. Emydina. Pedes plantigradi, digitis distinctis, plerumque palnatis: os corneum : collum retractile : pelvis mobilis. 
12. Testudinima. Pedes digitigradi, clavati, digitis indistinctis: os corneum : collum retractile: pelvis mobilis.

\section{Sectio 3. REPTILIA.}

Ordo 5. SAURII.

8. GeкKonide. Lingna brevis, crassi, papillosa, apice obtuso vix emarginata : oculi grandes, palpebris brevissimis haud conniventibus, posteriore obsoleta ; pupilla elliptica, verticali : os parietale dıplex : corpus depressum.

13. Gekkmine. Dentes maxillarm lateri interno adnati: aures conspicue, membrana profundata: squame dorsi parvule, tuberculis permixtis : digiti ]iberi, subrequales. Tarda: nocturna.

9. Stellonidæ. Lingua brevis, crassa, papillosa, apice obtuso vix emarginata: oculi palpebris comnjentibus clausiles, pupilla rotunda: os parictale simplex: corpus depressmm, dorsi culmine subplano, plemumque non cristato.

14. Agomina. Dentes adnati (maxillarum latere interno affixi).

15. Stelliomina. Dentes innati (maxillarum culmini comnati).

10. IgUANide. Lingua brevis, crassa, papillosa, apice obtuso vix emarginata: oculi palpebris conniventibus, pupilla rotunda : os parietale simplex: corpus plus minus compressum, in dorsi culmine carinatum vel cristatum.

16. Igumina. Dentes adnati, laniarii nulli.

17. Drucomina. Dentes innati, laniarii distincti.

11. C'Hsmeleontid.e. Lingua longa, carnosa, cylindracea, vibratilis, apice incrissato, integra, basi vaginata: gula dilatabilis: palpebre eirculares, foramine parvo, pupilla rotunda: corpus compressum.

18. Chamrelemtina. Dentes cum maxillis concretis: aures latentes: os frontale simplex: squamæ graniformes: cauda prehendens: pedes pentadactyli, digitis in duos oppositos fasciculos coadunatis.

1.2. Varanide. Lingua longissima, lavis, ingusta, vibratilis, longissime bifurca, basi raginata: liminic suprarbitales cutacce, ossiculo sn- 
perciliari accessorio : caput superne clypeolato-squamosum, pyramidale: corpus elongatum, depressiculum.

19. Varanina. Dentes adnati : os frontale duplex : cutis reticulatim exarati: pori femorales nulli : digiti liberi, inæequales.

13. Helodenatidæ. Lingua ...... : laminæe supraorbitales cutaceæ: oculi palpebrati: aures conspicuæ; membrana tympani superficialis: caput tuberculato-squamosum, depressum : corpus elongatum.

20. Helodermatina. Dentes adnati: cutis sulculis cxarata: squamæe tuberculiformes ossex: pori femorales nulli.

14. Aneivide. Lingua elongata, emissilis, squamuloso-papillosa, angusta, longissime bifurca: aures conspicure, membrana tympani superficialis : oculi palpebrati : laminie supraorlitales omnino cutacea : caput pyranidale, regulariter scutellatum.

21. Ameivina. Dentes adnati, corona posteriorum denticulata.

2.2. Podinemina. Dentes imnati, corona omnium simplex.

15. Lacertide. Lingua brevicula, squamuloso-papillosa, bicnspis: oculi palpebriti : laminæ supraorbitales subosseæ: squamæ difformes.

23. Lacertina. Dentes adnati: cutis flexilis: caput superne scutatum: cauda elongata, teres, verticillata: digiti simplices, subtus læeves.

24. Psammodromina. Dentes adnati : cutis flexilis: caput superne scutatum : cauda elongata, teres, verticılata: digiti, vel ad latera serrati, vel subtus carinati.

16. Ophiosauride. Lingua brevis, squamuloso-papillosa, apice attenuato obtuso plus minus excisa: oculi non semper palpebrati: aures conspicuæ: squanæ fasciatim posite, carinatæ: pedes in pluribus duo vel mulli:

25. Chamcesaurina. Dentes adnati: cutis rigida: squamæ angustæ, acute, in abdomine dorsoque æequales.

26. Ophiosanima. Dentes adnati: cutis rigida: squame subquadratie: plicatura lateralis.

17. Avguide. Lingua brevis, squamuloso-papillosa, apice attenuato obtuso plus minus excisa : ocnli non semper palpebrati : squimie uniformes, imbricatre, levigatie: pedes in pluribus cho vel nulli:

27. Scincina. Dentes adnati: cutis rigida. Habitus lacertinus: pedes 
quatuor, pentadactyli: aures conspicux; tyupani membrana profundata.

28. Anguina. Dentes adnati : cutis rigida. Habitus serpentinus: corpus cylindaceun, gracile: cauda longissima: artus, rel quatuor brevissini, renotissimi, vel posteriornm rudinenta tantum rel nulli:

18. Trphlopide. Lingua longa, bifurca: oculi vix ulli: squanze uniformes, imbricatre, lievigate: perles nulli.

29. Typhlopinu. Dentes adnati : cutis rigida. I Inbitus amplisbreninus: corpus longulum, cylindraceun, in utroque apice obtusum: cauda blevissima.

\section{Ordo 6. OPHIDH.}

19. ERrcide. Dentes rencnati unlli ; omnes breves, conici : calcarium rudimenta ad anum vix conspicua; pedum nulla: caput a trunco non distinctum, parvum, obtusum, scutis parum conspicuis: os parrum : oculi exigui: nares angustæ: corpus exile, undique cylindracemm : caula brevis, conica.

30. Eryciuu. Corpus graciliculum: squamæe cxigure, subrotundae, per series longitudinales dispositie: abdomen et canda subtus scutis simplicibus, hexagonis, transversis.

31. Calamerina. Corpus funicnliforme: squana prismatica, laevissina: abdomen et cauda subtus scut is parum numerosis.

20. Bolds. Dentes venenati nulli: calcaria cornea ad anmm: ossa interna pedum posticorum rudimentaria : corpus longissimmm, medio incrassatum ; camba teres, prohendens: caput a trunco distinctum, rasssum: oculi parvi, pupilla horizontali : nares fere supere: scuta abdominis candaeque inexpleta: squamie nmmerosie.

32. Boina. Intermaxillare edentulum : orbitie normales, ossibus frontalibus mediis superrne marginate: scuta abdominatia.

33. Pythomina. Intermaxillare dentatum : orbitae ab osse peculiari supranumelario perfectze: scutella abdominalia.

21. Acrochondus. Dentes venenati nulli: calcaria nulla: corpus undique squamosum, compressum: cauda compressa, valde prohendens. Aquutick.

34. Acrochomlina. Caput rotundatum: oculi exigui : nates superre, ap- 
proximatæ, tubulares: squamæ minimæ, non imbricatæ, mucronatæ: abdomen longitudinaliter squameo-carinatum.

22. Colubrids. Dentes venenati nulli : anus appendicibus destitutus: capnt scutis novem plerumque protectum : oculi naresque laterales: abdomen latissime scutatum : cauda teres.

35. Colubrina. Corpus fusiforme: caput latum : cauda modice elongata : squamæ in lineas longitudinales positæ. Terrestria.

36. Dipsadina. Corpus longissimun, graeillimum : eaput latum : cauda valde elongata: squanæ in lineas longitudinales positæ. Arborea.

37. Dendrophilina. Corpus longissimum, gracillimum: caput longum: cauda valde elongata: squamæ in lineas transversas positæ. Arborea.

38. Natricina. Corpus breviculum, torosum; abdomine dilatato, conrexo: eaput latissimnm, valde distinctum, conicum; rostro brevi, oris angulo elevato: cauda brevis: squame grandiculæe, carjnate, in lineas longitudinales positæ. Aquatica.

2.3. Hydride. Solidi dentes, venenatique in maxilla: cauda compressissima, remiformis. Marina.

39. Hydrina. Caput parvum, indistinctum: oculi naresque valvulares superi : scutula ventralia.

24. Najide. Venenati dentes, solidis sepius adjunctis, in maxilla: maxillare protractum : caput scutis tectum : oculi mediocres, pupilla rotunda: nares laterales, patulæ: corpus elongatum: cauda brevis, crassa, conica: squama grandes, rlomboideæ, plerumque lieves.

40. Bungarina. Collum haud dilatabile: caput elongatum, parum distinctum: corpus undique cylindraceun: cauda robusta: squame latæ, læeves, in lineas cireiter sexdecim positie.

41. Najina. Collum dilatabile: caput conicum, distinetum : corpus medio inerassatum : cauda elongata, conica: squame lanceolate, sepius carinatæ.

25. VIPERIde. Maxilla venenatis tantum dentibus armata: maxillare contractum: caput valde distinctum, depressum, postice dilatatum, squamis plerumque tectum: rostro truncato ac sape etian simo; 
labium superum prolapsum ; rictus arcuatus: oculi parvi, cavati, pupilla verticali : corpus abbreviatum, crassum : cauda brevissima: sfuamie lanceolatie, carinatre.

42. Crotulina. Fover pracoculares bina.

43. I iperina. Fovere prxoculares nullie.

\section{Ordo 7. SAUROPHIDI.}

26. Chalcrdide. Pedes quatuol.

44. Chalcidina. Truncus utrinque sulcatus.

27. Chenrotide. Pedes duo, antici: sternum ossiculo scapulo-claviculari utrinque, sine furcula.

45. Cheirotina. Dentes maxillis adnati.

28. Anphisberide. Nec pedes, nee apparatus sterno-scapularis.

46. Amphisbenina. Dentes maxillis adnati.

47. Trogonophina. Dentes cum maxillis concreti.

\section{Subclassis 2. DIPNOA.}

\section{Sectio 4. BATRACIIA.}

Ordo 8. BATRACHOPIDII.

29. Ciecrudes. Pedes mulli.

48. Cacilina. Cranium non suturatum : lingua mento tota affixa.

Ordo 9. RAN.E.

30. Rande. Ecaudata : corpus breve, latum : artus antici breviculi : sterum et claviculie perfectie: costae nulle: anus rotundatus. Lave upoda, candata, et corneo-rostrata: herbirora.

49. Pipina. Lingua sub cute absconclita: una tantum apertura pro tubis eustachianis.

50. Rumina. Lingua conspicua: tubse eustachiance distinctre: dentes maxillares: apices digitorum simplices.

51. Myladina. Lingua conspicua: tube enstachianae distincta: dentes maxillares: apices digitormm disciformes. 
52. Bufonima. Lingua conspicua: tubæe eustachianæe distinctæ: dentes nulli.

31. Salamandride. Caudata: corpus elongatum, subteres: artus æequilongi : sternum et clariculie nullæ: costæ: anus longitudinalis. Larea tetrapoda.

53. Pleurodelidina. Oculi congrui, palpebrati: appendix cutanea trunci nulla: cauda teres: costre verre.

54. Salamundrinu. Oculi congrui, palpebrati; appendix cutanea trunci nulla : cauda aut teres, aut compressa : costre veræ nullæ.

55. Andriadina. Oculi minimi, palpebris nullis ; appendix cutanea trunei utrinque natatoria: cauda depressa.

\section{Ordo 10. ICHTHYODEI.}

32. Anphunmos. Branchice obsoletre in respectiva cavitate latentes, foro externo utrinque laterali : cranium non suturatum.

56. Protonopsilina. Corpus granosum: rostrum productum: oculi minimi : cauda compressa : pedes quatuor, antici subpalmati.

57. Amphinmina. Corpus subteres: rostrum truncatum : oculi mediocres: cauda compressa : pedes quatuor, imbecilles.

33. Sirfixide. Branchiæ conspicuce, liberæ: cranium suturatum.

58. Hypochtomina. Pedes quatuor.

59. Sirenina. Pedes duo.

\section{CIAASSIS IV. PISCES.}

Animalia rertebrata sine pulmonibus, branchiis respirantia, sanguine frigido, rubro, ovipara vel ovovivipara, natantia: cor uniloculare, uniauritum: dentes fere in omnibus: corpus vel squamosum vel tubereulosum vel nudum: collum uullum : pinnæe loco artum. 


\section{Ordinum Distributio*.}

\section{Subclassis 1. ELASMIOBRANCHII.}

Branchiar fixa, haud operculata, lamellares, radiis verticalibus paucis rarisque superextensam membranam mucoso-vascularcm minute plicatam sustinentilus : cranium non sutusatum. Copula gaudent.

\section{Sectio 1. PLAGIOSTOMI.}

Sceletum cartilagineum, granulosum : ossa maxillaria ct intermaxillaria connata : cartilagines labiales in pluribus: dentes maxillis non infixi, sed cute tantum adjuncti, cum eaque mutantes: os transicrsum, latum : corpus aut tuberculatum aut nudum.

1. SElacha. (Chondropterygii.) Branchia penitus fixa: fissuris utrinque $5-7$.

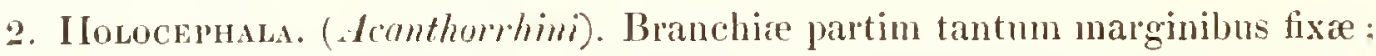
foraminibus quinque intcrioribus in fundo fissurie utrinque nnica: operculo tintum abortivo, sub cute latente: maxilla cum cranio connatit.

\section{Subclassis :2. LOPIIOBRANCHII.}

Branchia libera, palmiformes, radio verticali uno palmato in singulis arcubus: operculum unicum magnum membrana undique obseratum, parvo tantum juxta nucham formine relicto: cranimm suturatum.

\section{Sectio 2. SYNGNATHI.}

Sceletum fibroso-ossemm: maxillie perfecte, libcre.

3. Osteonerni. (Interopteri.) Corpus loricatmm, angulosum.

* Etsi ordinatorias meas hasce elucubratiunculas non despiciam, in quibus naturalem universorum Piscium harmoniam magis studueram consectari, fallacesque inde vel a sceleti compagine vel a pinnarum radiorum sirc molli sive rigida qualitate, vel a squamarum structura primarias definitiones desumptas magis magieque horruissem; non ideo tamen ubique quod penitius optaveram attigisse puto, rationemquc, non inepte fortasse, repcto a reverentia mea veteri erga clarissimos in philosophia naturali viros, inter quos principaliorem cathedram sibi merito praripuit Cuvierus, quem honoris causa nomino. Re tamen vera, ne sensibiles naturæe affinitates interciclerentur, Labrides, MHgilides, aliosque Acanthopterygios, Cyprinis addidi, Ophidides et Murenides a Scombris derivando. Ab Agassizianis denique, Cuvierianisque characteribus, quos ipse conserui discedendum magis forte erit quatenus Pissium ordines describi naturaliter velint. 


\section{Subclassis 3. POMATOBRANCHII.}

Branchiæ libera, operculatæ, radiis verticalibus numerosis in formam pectinis compositis, horizontalique lamellularum duplici serie infra supraque pectinulatis : cranium suturatum.

\section{Sectio 3. PLECTOGNATHI.}

Sceletum fibroso-cartilagineum: maxillæ imperfectre, non liberæ: opercula sub cute latentia; fissura branchialis utrinque parva.

4. Sclerodermi. (Acanthopteri.) Dentes distincti.

5. Grunodontes. (Pelropteri.) Rostrum corneum intrinsccus laninosum, loco et vice dentium.

\section{Sectio 4. MICROGNATHI.}

Sceletum cartilagineum, granulosum, processubus transversis osseis: vomer cum cartilaginibus frontalibus protractum : maxilla parra rudimentaria.

6. Sturiones. (Acipenseres.) Os labiis conosis cxigum, retractile.

\section{Scctio 5. TELEOSTOMI.}

Sceletum fibroso-osseum: maxillæ perfectæ, liberæ: corpus plerumque squamosum.

7. Ganomer. (Silmri.) Squama cortice vitreo, stratis infra lamellaribus, integris vel denticulatis subpositis.

8. Ctenoider. (Perce.) Squama asperæe, margine postico ciliato, stratis lamellaribus denticulatis subpositis.

9. Crcloider. (Cyprini.) Squamæ læeves, stratis lamellaribus integerumis subpositis.

\section{Subclassis 4. MARSIPOBRANCHII.}

Branchiæ fixæ, haud operculate, bursiformes, radiis vix ullis, membrana mucoso-vasculari superextensa contectis: cranium non suturatum.

\section{Sectio 6. CYCLOSTOMI.}

Sceletum membranaceo-cartilagineum: maxillæ connatæ: dentes non infixi: corpus nudum.

10. Hecminthoidei. (Lampetro.) Os ammulare, carnoso labio suctorio. 
Coxspectus Famliarum et Subfamiarum.

Subclassis 1. ELASMOBRANCHII.

Sectio 1. PLAGIOSTOMI.

Ordo 1. SELACIIA.

1. RaJnde. Cartilago peculiaris interior a nasali parte cranii ad principiun pinne pectoralis descendens: palpebrie aduatie: corpus depressun, dilatatum, plerumque nudum, pinnis amplissimis pectoralibus inclusum: analis mulla: bıanchiarum fissura intere.

Rajider revi. Corpus in forman cande abrupte defieiens.

1. Cephulopterimi. Capnt truncatum, appendicibus utrinque foliaceis tanquan bicone: pinuce pectorales latissima, in longissimos apices prodnctie : eauda tenuissina, clongatis. pinna dorsali et aculeo utrinque selrato munita: dentes minuti serratim positi.

2. Myliobutini. Caput ovatum, liberum a pinnis pectoralibus latissinis acuminatis: cauda tenuissima, chongata, pinna dorsali ct valido aculeo utrinque sermato armata: dentes grandes, complanati, tescellati ad instal operis musivi.

3. Anacanthini. Caput pimnis peetomalibus amplis precinctum : caucia tenuis, nee dolsali pimna nec aculeo munita: dentes minnti, tesselliformes, in quincuncen positi.

4. Trygonimi. Caput pimis pectoralibus amplis praccinctum : canda tenuis arnleo valido utringue serrato armata: dentes minnti, tesselliformes, in quincuncem positi.

5. Rajini. Corpus rhomboidale: caput pimis pectoralibus amplis propcinctun : cauda tenuis, elongata, pinnis dorsalibus duabus: dentes exigui, muncrosi, polymorphi, in quincunecu positi.

Rajida anomali. Corpus in formam eaudie garalatin deficicus.

6. Torpedinimi. Corpus orbiculare, laeve, caput pinnis pectoralibus anplis pracinctum : cauda crassa, ad basim depressa, mediocriter Jonga, 
pinna apicali ingenti, triangulari: dentes mimuti, acuti : apparatus electricus a capite inter branchias pinnasque pectorales.

7. Rhinobutini. Corpus rhomboidile elongatum, rostratum : cauda crassib carnosa: pinne dorsales duo, remotre: caudalis apicalis: dentes minuti, tesselliformes, in quincuncem positi.

s. Pristidini. Corpus elongatum, antice depressum: rostrum longissimum, planum, osscum, utrinque spinatum: dentes granuliformes. in quincuncem positi.

2. Squalide. Cartilago peculiaris interior prorsus nulla: palpebre liberie: corpus elongatum, subteres, siepius tuberculosum; tuberculis squamiformibus, minutis, plerumque integris, subovatis: pinne pectorales mediocres: brunchiarum fissure literiles.

* Siqualidre anomali. Corpus depressum : pimne pectoraies ampliculie.

9. Squatinini. Spiracula: pimna analis bullit.

* Squalidre veri. Corpus clongatum, teres: pinne pectorales congrnie.

10. Spinacini. Membrana nictitans nulla: spiracula: pinnce dorsales antice aculeat: ; analis nulla: dentes compressi, secantes; mandibulares cultro horizontali, margine laterali interno integro, externo cuspidato: tuberculi squamiformes tricuspides.

11. Scymmini. Membrana nictitans nulla: spiracula: pinnce dorsales inermes; analis nulla: dentes triangulares, maxillares lesiniformes, pluriseriales; mandibulares basi lata, mi- vel bi-seriales.

12. Notidanimi. Fissurie branchiarum utrinque ultra quinque: menbrana nictitans mulla: spiracula minima: pinna dorsalis mica; anilis: dentes mandibulares conjuressi, latissimi, pectiniformes; maxillares falciformes, apicibus ad angulum oris reclinatis: tuberculi squamiformes tricuspides: lingua adnata?

13. Triglochidini. Membrana nictitans nulla: spiracula parva: branchiarum fissure maximre, ommes ante pinnas pectorales sitie: pinnæe grandiculie; intilis; candalis lobo superiore clongato, superius minime excarata: dentes longi, acuti, utrinque denticnlo nno vel bino, 
14. Lammini. Membrana nictitans nulla: spiracula exigua: branchial'um fissurx maxima, ommes ante pinnas pectorales sita: secunda dorsalis et analis parve, oppositre; caudalis lunata, linc inde carinata, superius excarata: dentes sæpius acuti: valvula intestinalis spiralis.

15. Alopialini. Membrana nictitans nulla: spiracula minima: buanchialum fissur'x parva, ultima supra pectorales sita: dentes simplices.

16. Squalini. Membrana nictitans! spiracula nulla vel parva: fissuræe branchiaum ultima et sape etiam penultima supra pimnas pectorales sitre: dorsalis secunda anali subopposita: dentes compressi, triangulares, acuti.

17. Mustelini. Membiana nictitantis rudimentum: spiracula magna: pimme dorsales inermes; secunda anali opposita: dentes parvuli, obtusi, in quincuncem positi.

18. Cesteaciontini. Membrana nictitans nulla: spiracula grandicula: fissurae branchialum parve, ultima supra pectorales sita: dorsales antice aculeatre; analis: maxille exporrectre: dentes in quincuncen positi, mediocres, parvi, acuti, laterales latissimi.

19. Tricenodontini. Menbrana nictitans: spiracula vel nulla vel nediocria : branchiarum fissura ultina, et sæpe etiam penultima, supra pinnas pectorales sitee: secunda dorsalis anali opposita: dentes acuti, denticulis utrinque muniti.

20. Scyllini. Membrana nictitans nulla: spiracula amplicula: pinnae dorsales anbae ventralibus non anteposita: dentes parvi, acuti, denticulo utrinque uno vel pluribus: tuberculi squamiformes tricuspides: valvula intestinalis spiralis. Oripari!

\section{Ordo 2. HOLOCEPHALA.}

3. ('Hundride. Corpus laeve, vel exiguis aculeis hispidum: dura loco dentium scuta, supra quatuor, infia duo.

21. Chimerini. Rostrum prominulum, antice foliaceum: pinnarum dorsalium prior valido aculco armata. 


\section{Subclassis 2. LOPHOBRANCHII.}

\section{Sectio 2. SYNGNATHI.}

Ordo 3. OSTEODERMI.

4. Syngnathide. Corpus scutatum.

22. Pegasini. Os inferum ad basim rostri.

23. Syngnathini. Os terminale in apice rostri.

\section{Subclassis 3. POMATOBRANCHII. \\ Sectio 3. PLECTOGNATHI. \\ Ordo 4. SCLERODERMI.}

5. Balistididæ. Rostrum conicum, productum: os exiguum.

24. Balistidini. Corpus compressum, squamis magnis durissimis rhomboidalibus tectum.

25. Ostraciontini. Corpus polyedrum, cataphractum.

Ordo 5. GYMNODONTES.

6. Tetraodontide. Colpus inflabile, plus minus spinosum.

26. Tetraodontimi. Maxilla utraque rostri bipartita, vel saltem superior. marginibus obtnse denticulatis: aculei corporis breves.

27. Diodontini. Maxilla utraque rostri simplex, integra: aculei corporis longi.

7. Orthagoriscide. Corpus non inflabile, haud spinosum: sceletum fere omnino cartilagineum.

28. Orthagoriscini. Corpus admodum compressum, postice truncatum, pinna candali profunda, brevi: ventralibus nullis : anali valde retroposita.

\section{Sectio 4. MICROGNATHI.}

Ordo 6. STURIONES.

8. Polyodontuda. Corpus læve: dentes minuti, numerosi.

29. Polyodontini. Rostrum longissimum, dilatato-foliaceum: opercula longissimo mucrone munita. 
9. ACHENSERIDa. Colpus scutatum: dentes nulli.

30. Lcipenserim. Rostrum mediocriter longum, modice angustum : opercula inermia.

\section{Sectio 5. TELEOSTOMI.}

\section{Ordo 7. G.LNOIDEI.}

10. Loricarid.:. Corpus scutis osscis tectum: os inferum : opercula branchialia inmobilia: ossa intermaxillaria parva, maxillaribus transversis lauml contignis: prinus radius pinnarun dorsalis, pectoraliun et ventıalium valielus, aculeatus; cateri unolles.

31. Loricarimi. Pinna dorsalis mica, secundal quandoque exigua.

11. Sılurud.e. Corpus nudum : ossa intermaxillaria maxima, marginem maxille constituentia, maxillaribus subabortivis, ant in cirros conversis: pinma dorsalis postica plerumque adiposa: radius primus dorsalis inticie atque pectoralium passin aculeatus, articulatus, caeteri molles: piunce ventrales infia pectorales site: os terminale: opercula branchialia nobilia.

3:2. Cullichtimi. Os parrum; dentes fere inconspicui : corporis nudi series laninamun quatuor ad latera: pinnie dorsales duo, secunda radio inicu.

33. Pimelodimi. Os modicum; dentes forma et loco varii : latera vel inermia, vel una tantum laminarum serie: pinnse dorsiles duo, secunda atliposis.

34. Silmimi. Os amplum; dentes numcrosi: corpus omnino nudum : pinna dorsalis mucal, exjgna ; analis longissina.

12. Lepidostende. Corpus squanis lapieleis: omnes pinnarum radii molles: pinnie rentrales post pectorales ossibus pelvis non appensie.

3.j. Lephlosteimi. Rostrum intermaxillaribus, maxillaribus, palatinisque ossibus cum romere atque ethmoideo connatis: menbrana branchiostega trilatdiata: dorsalis pinna ac analis pariter et valde retroposite: radii pinnamum primi squanco-semati.

36. Polupterimi. Maxilla margine immolsili, intermaxillaribus mediis, maxillaribus litteralibus: membrana branchiostega uniradiata : pinne floriles numerose, singulae aculeo munitre. 
13. Tetragonuride. Corpus squamis grandibus, asperis, ciliatis, cuti adhærentissimis: dentes validiculi, acuti: pinne dorsales dno; antica radiis spinosis longa, humilis; ventrales paulo post pectorales.

37. Tetragomurimi. Canda utrinque cristata! labium inferius intus carinatum.

14. Macrouride. Squamæ duriter asperæ: os inferum ! pinnæ ventrales infra gulam sitæ, acuminatæ: omnes pinnarum radii molles.

38. Macrourini. Corpus elongatum, tereticulum, postice compressum, attenuatum, antice rostratum: os infermm: pinna dorsales duo; postica elongata eandalem acuminatam cum anali conjunetam at tingens.

\section{Ordo 8. CTENOIDEI.}

15. Pleuronectide. Corpus eximie compressum: caput non symmetricum : oculi unilaterales: radii omnes pinnarum molles: pinnæ ventrales sub pectoralibus: pelvis ossibus humeralibus appensa.

39. Soleim. Maxilla longior mandibula : "oculi parvi : nares unilaterales: præoperculum ab opereulo non distinctum: pinnæ pectorales parvulæ aut nullie: squanæ laminula pedunculatæ: linea lateralis recta.

40. Pleuronectini. Mandibula longior maxilla: oculi grandes: nares hinc inde binæ: laminæ operculares distinctæ: pinnæ pectorales congruæ: squamæ sessiles: linea lateralis antiec arcuata.

16. Снетоdontide. Corpus compressum : palatum edentulum : dorsalis pinna atque analis aeque ac in trunco squamis magna saltem ex parte ob. tectæ, radiis spinosis pluribus; ventrales unico.

41. Pimelepterimi. Dentes sectorii.

42. Chcetodontini. Dentes setacei, conferti in utraque maxilla. Colores admodum vivi.

17. Anabantide. Celhulæ in pharyngeis ossibus superioribus ab eorum foliolis irregularibus dispertitæ! Radii pinnæ dorsalis et analis plures spinosi. Diu extra aquam vivere valent.

43. Anabontini. Corpus teres : caput latum, rostro brevi, obtuso: squamæ solidæ: linea lateralis interrupta. 
18. Fistularidæ. Rostrum tubuliforme ore exigno terminali: radii spinosi plures in prima saltem dor'sali.

44. Coproidini. Colpus sublotundum, compressum: rostro brevi.

45. Centriscini. Corpus ovale, compressum : rostro longissimo.

46. Fistularimi. Corpus elongatum, cylindraceum : rostro longissimo.

19. Monida. Os valde protractile: palatum dentibus instructum, vel (præeopereulo margine (lenticulato) edentulum: radii spinosi plures in pinna dorsali ac anali ; unus in utraque ventrali.

47. Momini. Pinna dorsalis non squamosa.

48. Casionini. Pinna dorsalis squamosa.

20. Sparide. Laminx operculares integrie, spinis carentes: os non protractile: palatum edentulum: squama grandes: pinna dorsalis squamis destituta, radiis spinosis pluribus ac in anali; ventrales unico.

49. Obladini. Dentes sectorii, uniseriales; molares bemisphrerici nulli.

50. Cantharini. Dentes numerosi, conferti, tenuissimi.

51. Lethrinimi. Dentes interdum molares liemisphierici, uniseriales: genæe sine squamis.

52. Denticini. Dentes omnes conici; nonmulli ex anterioribus producti, incurvi : gence squamose.

53. Sparini. Dentes molares hemisphaerici: genxe squamosie.

21. Chromidide. Laminae opereulares integrie, spinis carentes: labia carnosa duplicata: dentes in maxillis nec non in tribus ossibus pharyngeis: squama grandes: pinua dorsalis mica, radiis filamentose appendiculatis, spinosis pluribus ac in anali; ventrales unico.

54. Chromidimi. Corpus oblongum : dentes maxillares et pharyngei tenuissimi, conferti, preposita conicornm majorum serie.

55. Cychlimi. Corpus elongatum : dentes omnes tenuissimi, conferti.

22. ScreNide. Lamina operculares margine denticulatie aut spinosie: genæe non loricatæ: os protractile: vomel et ossa palatina dentibus destituta: pinne verticales sape squanosie, radiis spinosis pluribus; ventrales unico.

56. Pomacentrini. Cranium minime tumens, nec ossibus cavernosis constans: linea lateralis sub pinne dorsalis termino interrupta. 
57. Scicnini. Cranium tumens, ossibus cavernosis conflatum : linea lateralis continua.

23. Triglidx. Genæe loricatæ (lamina suborbitales genam utrinque partim obtegentes cum præoperculo connexæ): radii spinosi plures in pinnis dorsi et anali, in ventralibus unicus.

58. Cottini. Pinnæ dorsales dno: caput vel rotundatum vel depressum.

59. Scorponini. Pinna dorsalis unica.

60. Triglini. Pinnæ dorsales duo: caput parallelopipedum.

24. Mulud vum dentibus tenuibus : cirri duo sub mandibula retractiles: capitis et trunci squamæ late, facile decidure: ralli spinosi plures in pinna dorsali antica, unus saltem in anali, et in utraque ventrali.

61. Mullini. Pinnæ dorsales binæ distinctæ.

25. Percidx. Laminæ operculares margine denticulato aut spinoso: genæ non loricatie: dentes in maxillis, in vomere et fere semper in ossibus palatinis: pelvis ossibus humeralibus appensa: radii spinosi plures in pinnis dorsi et anali, in ventralibus unicus.

62. Polynemini. Pinne ventrales post pectorales : rostrum tumidum : pinnæ verticales squamosæ: radii pectoralium plures liberi, filiformes.

63. Holocentrini. Pinnæ ventrales, radiis plusquam quinque, sub pectoralibus: membranæ branchiostegæ radiis plusquam septem.

64. Percini. Pinnæe ventrales quinque-radiatæ sub pectoralibus: membranæ branchiostegæe radiis septem vel minus.

26. Goвids. Pinnæ ventrales infra pectorales ortæ, conjunctæ saltem ad basim in formam disci: radii spinosi pinnæ dorsalis graciles, flexiles: fissuræ branchiales parva.

65. Gobini. Corpus elongatum, parum compressum, antice incrassatum : squamæ minutæ. Appendix tubulosa conica post amum, in utroque sexu.

\section{Ordo 9. CYCLOIDEI.}

27. Cyclopteride. Pinne ventrales infra pectorales sitæ in discum suctorium conniventes: radii omnes pinnarmm molles: corpus nudum!

66. Cyclopterini. Corpus tumidum: pinnx pectorales jugulares latæe, membrana junctæ: operculum exiguum. 
28. Blennide. Pinma ventrales ante pectorales, distincta, didactyla: radii spinosi pinnae dorsalis graciles, flexiles.

67. Blemini. Corpus elongatum, compressun, mucosum. Appendix tubulosa conica post amm, in utroque sean.

29. Callyonnode. Pinne rentrales infra gulan insertæ, remotissima, pectoralibus ampliores: radii spinosi pinne dorsalis graciles, flexiles: apertura branchiali utrinque prope nucham exigna : corpus nudum !

68. Callymimini. Corpus vix complessum, antice incrassatum, osse tympanico postice elongato, aculeato: oculi superi, approximati. Appendir tubulosa conica post anum.

30. Lophids. Pinme pectorales perlunculata: apertura subtus branchialis utrinque cxigna operculis a cute contectis: radii anteriores pinnce dorsalis subspinosi : corpus nudum: sceletum fere cartilaginemu.

69. Loplimi. Pinna ventrales longula, humcro ante pectorales infixe, quinque-radiatie.

70. Butruchimi. Pinnxe ventrales sub gula infixa, angustre, tri-ladiatae.

31. Goddde. Pinnac rentrales sub gula infixie, acuminatae pelvis ossibus humeralibus appensa: squamie molles: radii ommes pinnarmm molles.

71. Runicepini. Caput latissinum, valde depressum : pinna dorsalis antica humillima, vix exserta.

72. Galini. Corpus modice abbreviatum, parum compressum: caput congrumm, vix mquam squamosum, cirlo unico vel nullo: squama minnte : pinnic dorsales vel duo vel tres ; analis sapius duplex; candalis sepius furcata.

73. Lotini. Corpus clongatum, hubricun, postice compressissimum : caput magnum squamosmm, cirro uno vel pluribns: pinna dorsalis ma vel dno; analis mnica; caurlalis integra, plus mimus rotundata.

32. Crprinide. Pinna adiposil et intestina caca mulla: maxilla margo ab intermaxillaribus ossibus constitutus: rictus modicus: maxille debiles edentule: ossa pharyngea dentibus validis, specificis: radii branchiales tres: pinne ventrales post pectorales: pelvis ossibus huncralibns non appensa: radii pinnarmm proprie spinosi mulli. Certeris piscibus mimus camicori. 
74. Cyprinini. Pinna analis imperforata: corpus mucosum, squamis profunde insitis, raris : os cirrosum.

75. Leuciscini. Pinna analis imperforata: corpus vix mncosun, squamis superficialibus densis : os non cirrosum.

33. Pecilid Pinna adiposa et intestina cæca nulla: maxillæ margo ab ossibus intermaxillaribus constitutus: rictus modicus: dentes in maxillis: radii branchiales plus quam tres: pinnce ventrales post pectorales : pelvis ossibus hmmeralibus non appensa : radii pinnarum spinosi nulli.

76. Amableptini. Pupillie duplices (vitta transversali cornean et iridem secante) : pinna analis apex perfolatns. Tivipuri.

77. Pcecilini. Maxillie depressæ, protractiles.

34. Labride. Labia carnosa, duplicata : corpus oblongum : squanæ grandes: pinna dorsalis unica, radiis spinosis membrana plerumque appendiculatis : pelvis ossibus humeralibus appensa.

78. Labrini. Dentes maxillarmu robusti, conici, inæequales.

79. Scarini. Dentes lamellosi, imbrieati.

35. Mugrude. Caput depressum, squamis latis, etiam polygonaribus, tectum : labia crassa, inferum intus carinatum : cirri nulli : opercula integra: squamæ grandes: pinnæ dorsales dno, radiis spinosis pluribus ac in anali; ventrales paullo post pectorales, radio spinoso unico.

80. Mugilini. Pinna dorsalis antica, radiis tantum quatuor: dentes tennissimi : squame simplices, decidne.

36. Atherinide. Caput pyramidale, eute lævi obtectum: os valde protractile: labia tenuia: dentes minutissimi: cirri nulli: opercula integra: squanæ tennes, translucidæ: pinnæ dorsales duo, remotissime, radiis spinosis pluribus ac in anali; ventrales longe post pectorales, radio spinoso unieo.

81. Atherinini. Corpus elongatum, faseia argentea longitudinali ad latera.

37. Ophiocephalide. Cellnla in pharyngeis ossibus superioribus ab eornu foliolis irregularibus dispertitæ! Radii pinnarum omnes molles: primus ventralinm simplex. Diu extra aquam virere valent. 
82. Ophiocephalini. Corpus elongatum, fere cylindraceun: caput depressum scutis polygonis tectum : rostrun breve, obtusum : radii branchiales quinque: dorsalis pinna ac analis longissinre; caudalis l'otundata: linea lateralis contimma.

38. Amp.e. Pimna dorsalis mica, radiata, longa: intestina caca nulla : margo maxillie ab intermaxillaribus ossibus in medio a maxillaribus in ramis constitutus: squame grandes: caput loricatum: radii pinnarum omnes molles: pelvis ossibus humeralibus non appensa.

8:3. Amimi. Pinna analis brevis: radii branchiales duodecim : nares brevi tubo appendiculatx: dentes antici, conici; postici tessellati : vesica aerea cellularis.

39. CLupeidex. P’inna dorsalis unica, radiata : intestina ceca plurina: margo maxillae ab intermaxillaribus ossilıns in medio, a maxillaribus in ramis constitutus: squamæe grandes: caput non loricatum: radii pimnarum omnes molles: pelvis ossibus lumeralibus non appensa.

84. Erythrichtrimi. Caput rotundnm, obtusum, minime squanosnm, ossibus duris: genxe a suborbitalibus duris ossibus protectx: pinna dorsalis ventralibus respondens: vesica aerea ampla.

85. Chupeini. Caput plus minus oblongum, acntulum, squamosmu : genæ simplices: venter plerumque compressissimus, seratus: branchiae late fixae: pinma dorsilis varia: vesica aerea longa, acuta.

10. S.muonme. Pinna dorsalis antica, radiis omnibus mollibus; postica parva, adiposa, minine radiata: intestina ceca plurina: corpus valde squanosum: pelvis ossibus lumeralibus non appensa.

46. Scopelini. Margo maxilla ab ossibus maxillaribus ex toto constitutus: rictus amplissimus: dentes minimi : lingua et palatum edentula: radii branchiales minus quam duodecim: squamre grandes, lieves, deciduce: pinna ventrales postpositre: pinna dorsalis postica fere semiladiatia!

87. Aulopodini. Margo maxillae ab ossibus maxillaribus ex toto constitutus: rictus amplissimus: dentes numerosi, acutissimi, vel in lingua et ossibus pharyngeis: radii branchiales plus quam duodecin : corpus nndique squanosnm: squamae grandes, adharentes, cilatæ: pinnce ventrales praposita. 
88. Salmonini. Margo maxille ab ossibus maxillaribus partim constitutus: rictus amplus: dentes sæpius acuti, serie unica vel duplici in ossibus maxillaribus, intermaxillaribus, palatinis, mandibularibus et pharyngeis, in vomere et lingua: squamæ parvulæ, integerrinze: pinuæe ventrales postposite. Ad hos spectant perfectissimi piscium quoad dentes.

89. Miletidini. Margo maxille ab ossibus maxillaribus partim constitutus: dentes obtuse prismatici, corona tricuspide: rictus parvus: lingua et palatum edentula.

90. Hydrocionini. Margo maxilla ab ossibus maxillaribus partim constitutus: rictus amplus: dentes conici vel acuti : vomer et lingua edentuli : genæ a lamina suborbitali protectæ.

41. Esocıde. Pinna dorsalis unica, radiata, retroposita, radiis spinosis nnllis ac in cæteris pinnis: intestina cæca nulla: maxillæ margo ab intermaxillaribus constitutus, aut maxillaria edentula occulta sub labiis: dentes in maxilla ac in longiore mandibula nonnulli acuti : corpus parce squanosum : pelvis ossibus humeralibus non appensi. Voracissimi.

91. Esocini. Pinnæ pectorales congruæ; dorsalis et analis breves, rotundatæ: corpus parce elongatum, cylindraceum, profundulum: squamæ duræ, grandiculæ: linea lateralis unica: rictus amplus: mandibulæ latæ, haud rostrate: margo maxille a vomere et ab exilibus intermaxillaribus anterius constitutus, in ramis a maxillaribus elongatis: dentes pluriseriales in vomere, palato et lingua; miseriales in intermaxillaribus et mandibula; pharyngeorum acuti.

92. Belonini. Pinnæ pectorales congruæ; dorsalis et analis longa, falciformes : corpus valde elongatum, gracile, subquadratum, lineis lateralibus duabus: squamæ raræ, tenues: rictus parvus: mandibulse longissimæ, angustæ, in rostrum acutum protracte: margo maxillæe ab intermaxillaribus unice constitutus: dentes infra supraque uniseriales; in palato et lingua nulli; pharyngeorum hemisphierici.

93. Exocetini. Pimm pectorales maximæ, volatui aptæ:

42. Sphyrenide. Pinnæe dorsales duo remotæe, radiis spinosis pluribus ae in 
anali; ventuales mnico: intestina caca plurima: dentes tantum in maxillis et in ossibus palatinis; canini plures valde arnti: corpus elongatum: lamina operculares integræ: pelvis ossibus humeralibus non appensa.

94. Sphyromini. Pinna dor'salis postica congrua.

95. Parulepidini. Pinna dorsalis postica exillina.

43. 'Trachinde. Pinna dorsalis unica, elongata, radiis spinosis phribus; unus saltem in anali et in utraque ventrali : dentes in maxillis, in romere et sape in ossibus palatinis: operculum aculeatum : pinna ventrales ante amplas pectorales: pelvis ossibus humeralibus appensil.

96. Trachinimi. Gena simplices.

97. Cramoscopini. Gence psendo-loricata (lamina suborbitales latissinæ, posterius connexie ossibus tympanicis, minime vero prieoperculo).

44. Teuthydde. Corpus compressum, oblongum : os parvum, non protractile: dentes sectorii in utraque maxilla uniseriales: palatum et lingua edentula: radii spinosi plures in pinna dorsali, unus saltem in anali et in utraque ventrali.

98. Teuthydini. Pinna dorsalis unica.

45. Echeneudna. Caput superne complanatum in disco ovali laminoso: pinnat ventrales infra pectorales: pelvis ossibus humeralibus appensa : pinnarum radii omnes molles.

99. Echeneidini. Corpus fusiforme, elongatum: squama vix conspicux: punna dorsilis anali opposita.

16. Mormyride. Corpus compressum, oblongum, squamosum: caput ultra opercula cute crassa olvolutum: os minimum: fissura branchialis parva, verticalis: intestina caeca duo: pelvis ossibus humeralibus non appensa : radii pinnarmu omnes molles.

100. Mormyrini. Pinna dorsalis mica.

47. Gasterosteide. Gence loricatre: (laminie suborbitales genas partim obtegentes cum prieoperculo connexie) : radii pinnarum aliquot aculeati : pelvis ossibus humeralibus appensa.

101. Gasterosteini. Aculei aliquot liberi loco pinnæ dorsalis antica.

4๖. Sconbrun. Corpus puasi leve, squamulis parvulis: lamine operculares 
integre: eauda robusta: pinnie verticales squamis plerumque destitutr, radiis spinosis pluribus; rentralibus unico.

102. Centronotini. Aculei aliquot liberi loco pinnæe dorsalis antica.

103. Aiphiadini. Pinna dorsalis unica, continua: rostrum ensiforme.

104. Carangini. Linea lateralis lorieata:

105. Bramini. Pinna dorsalis et analis æequis ac in trunco squamis partim obtectæ: corpus compressum: palatum dentibus avenatum.

106. Stromateini. Pinna dorsalis unica, elongata, post pectorales orta, radiis spinosis interdum mollibus: eorpus valde compressum: squanæe exiguie: capitis vertex subrotundus.

107. Coryphenini. Pima dorsalis unica, longissima, radiis spinosis intcrdum mollibus, dorsum unirersum fastigians: corpus cylindraceo-compressum, elongatum: squamæ exiguæ: capitis rertex acutus.

108. Zeini. Pinua dorsalis unica: corpus ralde compressum, vix squamulosum: os valde protractile.

109. Vomerini. Pinne dorsales duo: corpus ralde compressum, vix squamulosum: eapitis vertex anceps.

110. Scombrini. Pinna dorsalis antica contimua; postica in plures pinnulas spurias dirempta aque ac pars respondens analis: corpus fusiforme.

111. Trichurini. Pinna dorsalis unica, continua: aculei multi exigui, liberi, in locum pinnæ analis saltem partin: corpus prælongum, valde compressum : rostrum elongatum : os profunde fissum.

49. Cepolide. Corpus prælongum, valde compressum: squamæ minuti: rostrum breve: os parrum, parum ant oblique fissum : radii spinosi plures in pinnis dorsali ac anali, unus in ventralibus.

112. Cepolini. Pima dorsalis unica, longissima.

50. Ophididæ. Corpus ensiforme, lubricum: opercula manifesta: fissuræ branchiales grandes: squamæ parrulæ cuti intrusæ: omnes pinnarum radii molles: pinne rentrales uulle.

113. Ophidini. Rostrum obtusum, non extensile: pima dorsalis, analis, et caudalis acuta, omnes conjunetie.

114. Ammodytini. Rostrum acutum : maxilla extensilis ultra longiorem YOL. XVIII. 
mandibulam: pinna dorsalis longa; analis et caudalis bifurcæ, omnes distinctæ.

51. Murandde. Corpus præelongum, cylindraceum, lubricum: opercula parva, sub cute latentia: fissuræ branchiales minimæ: squamæ tenuissimæ, cuti intrusæ: pinnæ ventrales nullæ: ommes pinnarum radii molles.

115. Murcenini. Aperturæ branchiales tubulate.

116. Gymmonotini. Aperturæ branchiales ante pinnas pectorales, membrana partim tectæ.

I17. Symbranchini. Apertura branchialis foramine unico subgulari : pinne verticales subadiposæ.

18. Apterichtrini. Aperturæ branchiales subgulares proximæ: pinnæe vel fere, vel omnino nullæ.

\section{Subclassis 4. MARSIPOBRANCHII.}

\section{Sectio 6. CYCLOSTOMI.}

\section{Ordo 10. HELMINTHOIDEI.}

52. Petronyzonide. Corpus elongatissimum, cylindraceum, undum: pinne sine radiis.

119. Petromyzomini. Foramina branchialia ad colli latela utrinque septem.

120. Gastrobranchini. Foramina branchialia bina gularia. 



\section{$2 \frac{1}{2} a^{2}$}

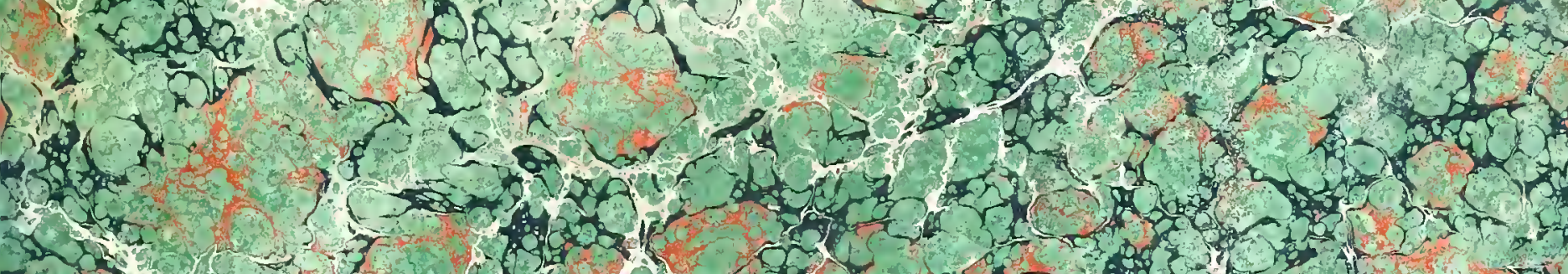

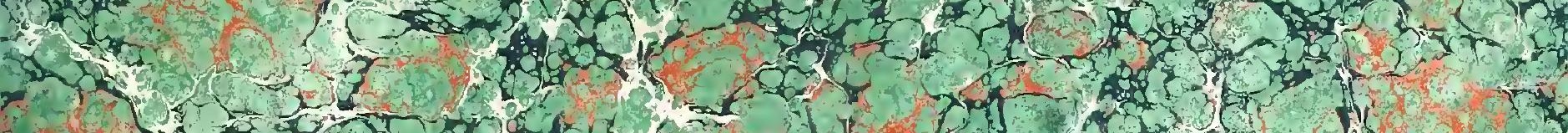

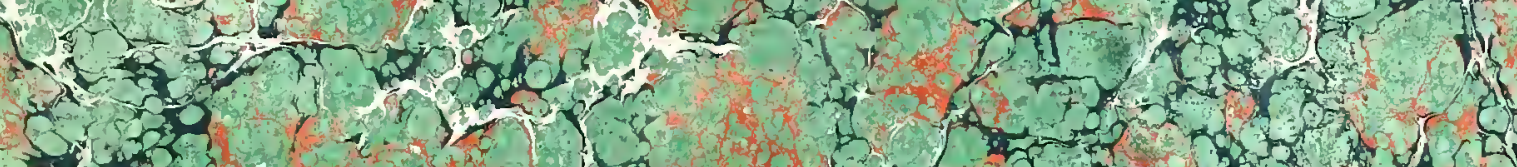

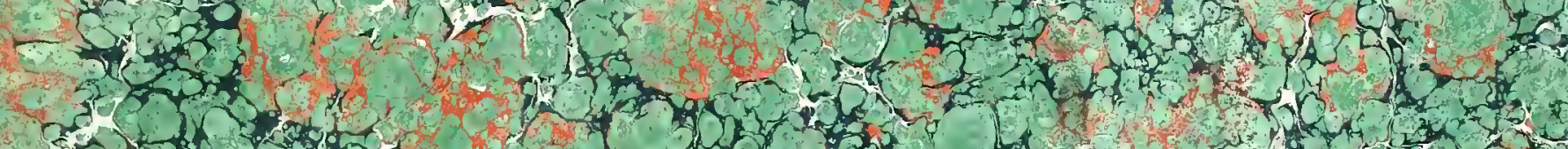

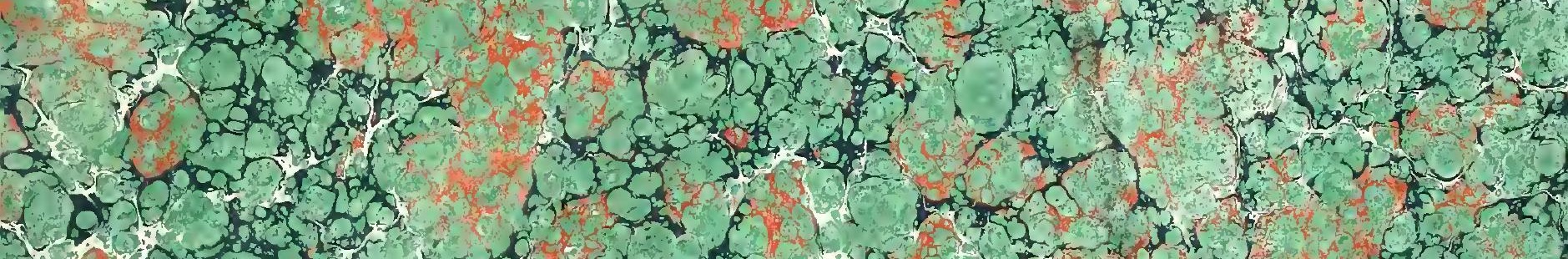

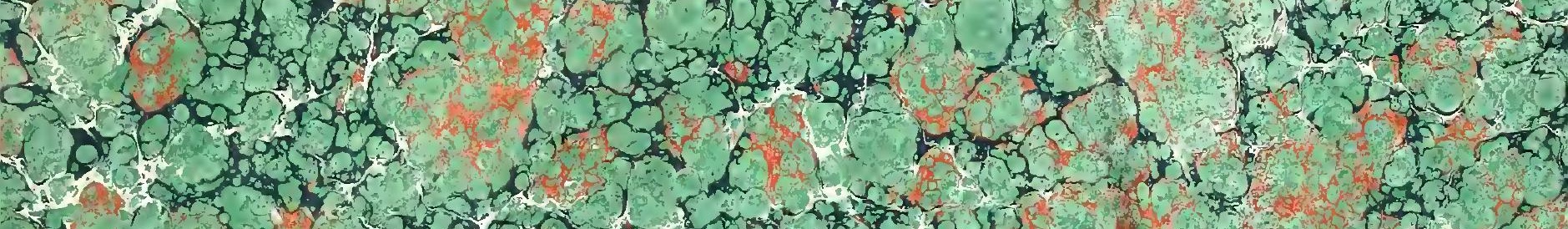

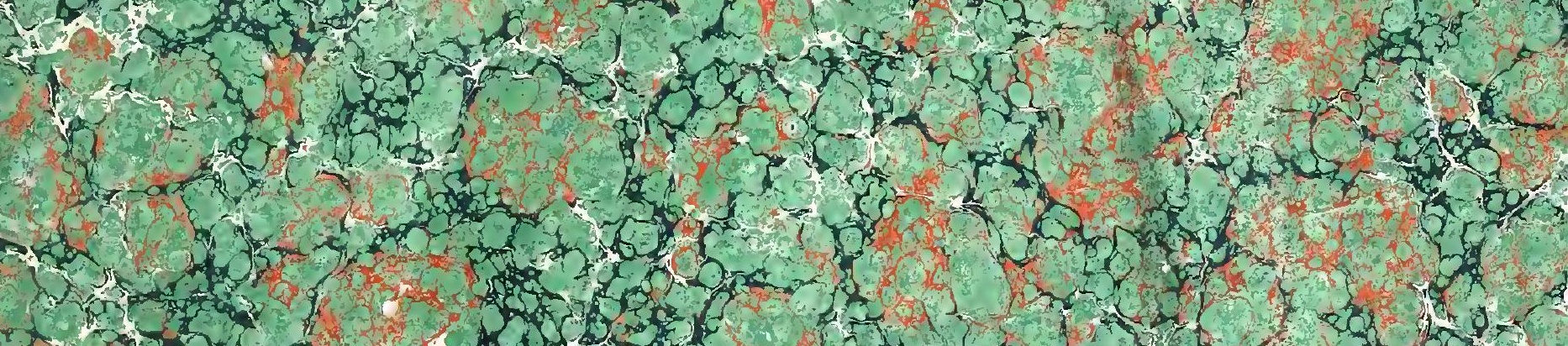

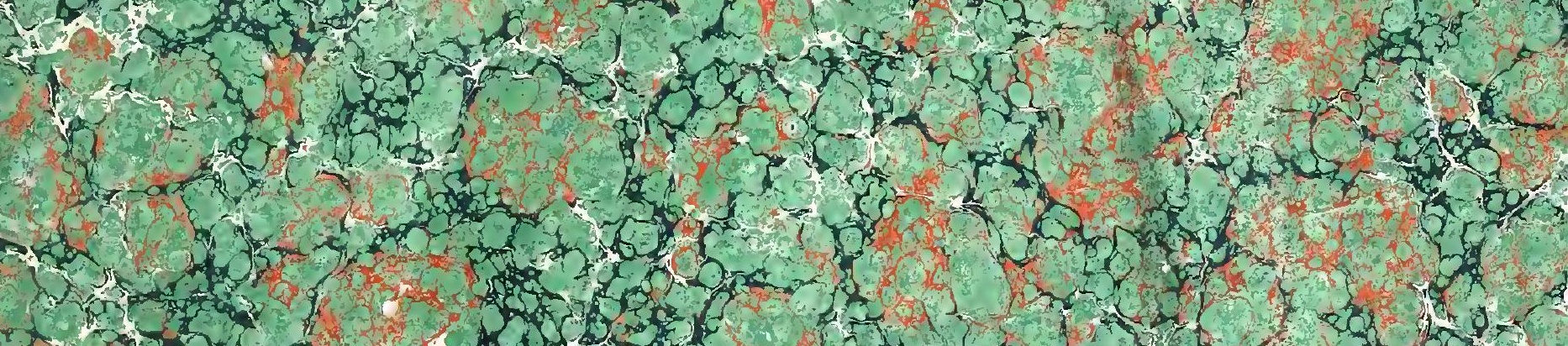

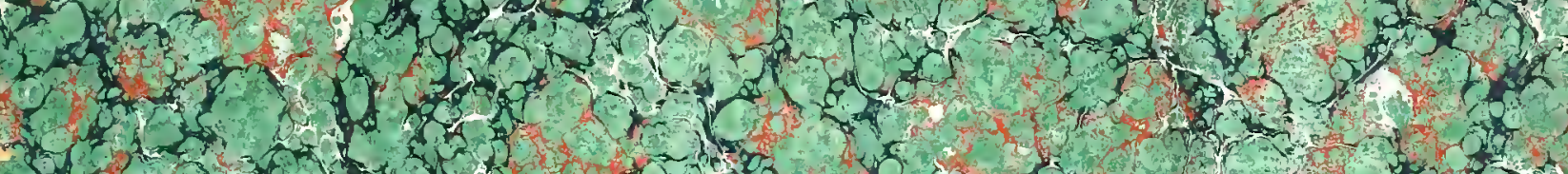

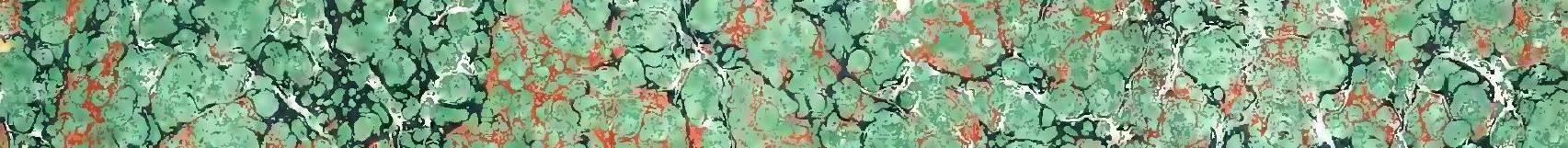
$x+3 x+2$ 7.

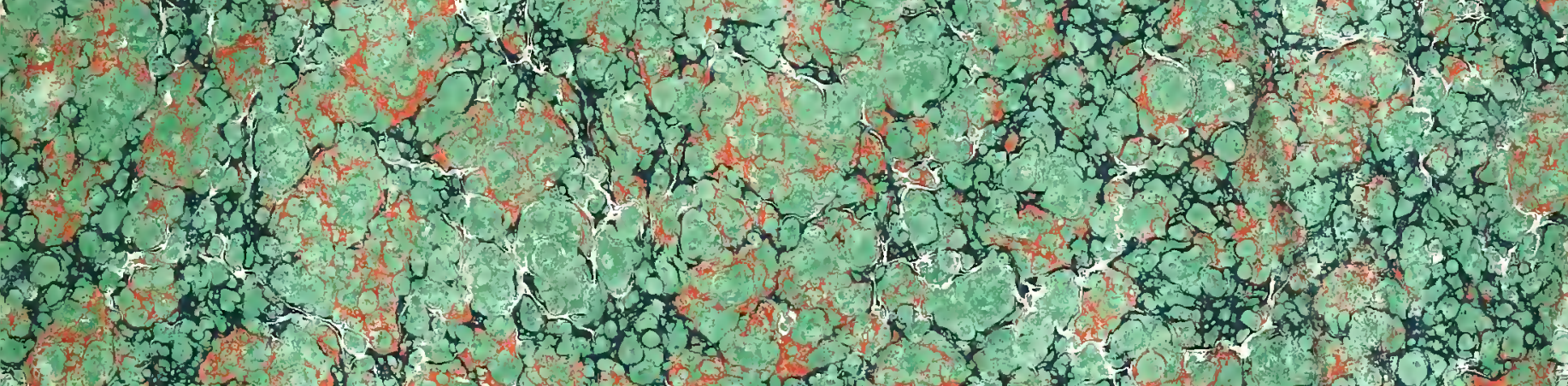


$7+$

\%

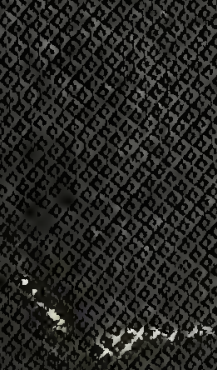

8,85

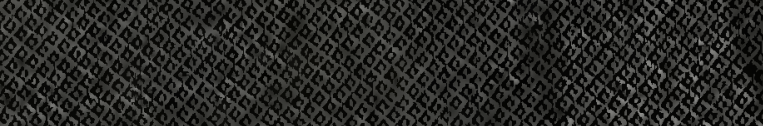

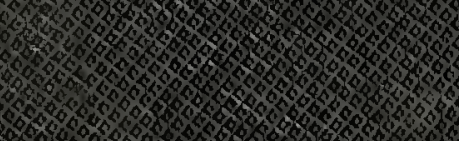

W.

so

y.

$8 \%$.

$8 \%$

得

\%

s.

r. 